\title{
Empirical Susceptibility, Vulnerability and Risk Analysis for Resilience Enhancement of Urban Areas to Terrorist Events
}

\author{
Georg Vogelbacher ${ }^{1}$ Ivo Häring ${ }^{1}$ Kai Fischer ${ }^{1}$ • \\ Werner Riedel ${ }^{1}$
}

Received: 4 August 2016/Accepted: 9 September 2016/Published online: 26 September 2016

(C) Springer International Publishing Switzerland 2016

\begin{abstract}
The worldwide progressing urbanization increases the need to consider threats like terrorism or natural disasters when planning or redesigning urban areas. The paper shows how to assess and mitigate terrorism risks in a systematic way using historical event data on urban plan level. Based on multiple event data, within a risk approach, quantities are derived that measure averaged susceptibilities, vulnerabilities and risks for buildings and infrastructure in the context of urban planning. The empirical approach allows for local scaling factors for frequency of events, e.g., due to physical accessibility, and for consequences, e.g., due to physical counter-measures. The paper discusses the input data necessary for statistically sound analyses, in particular, the accuracies of the quantities as well as deep uncertainties. To this end, it determines distributions for the frequencies and consequences, in particular, introducing a modified Pareto distribution. The application to a sample urban area demonstrates how the analysis results lead to a more resilient scenario using the analysis and selecting resilience improvement options. It is shown that the empirical analysis motivates more detailed additional engineeringsimulative approaches for resilience improvement through averaging susceptibility, vulnerability and risk assessments by assuming multiple spatially distributed events.
\end{abstract}

Keywords Urban planning · Susceptibility · Vulnerability · Risk · Terror event database · Resilience - Quantitative assessment

Ivo Häring

Ivo.Haering@emi.fraunhofer.de

1 Fraunhofer Institute for High-Speed Dynamics, Ernst-Mach-Institut, Am Klingelberg 1, 79588 Efringen-Kirchen, Germany 


\section{Introduction}

Security and safety issues become an increasingly important factor in modern urban planning. In 2007, the degree of urbanization reached a value of $50 \%$ and has an ever-rising trend (United Nations Department of Economic and Social Affairs 2014). A growing population density in cities and their agglomerations has a pronounced effect on their vulnerability to hazardous events (Cross 2001). Urban areas comprise the most critical infrastructures of the society and significantly specify their respective resiliencies (Branscomb 2006). As depicted by Branscomb (2006), cities become more vulnerable to three kinds of disasters: natural disasters (e.g., hurricanes, flood, earthquakes, tsunamis), technogenic disasters (resulting from human error and failing infrastructure, e.g., power failure) and terrorism. The present work has the focus on contributing to counter threats in urban environments based on the analysis of past events. It can be extended to all types of hazards for which similar empirical event data are available.

Terrorism is an asymmetric threat of growing importance which can pick targets anywhere (Lin et al. 2007). Savitch (2005) emphasizes that urban spaces have become prime targets and cities are more likely to be attacked. Terrorism events are often categorized as threats with low probability and potentially high consequences, for instance in FEMA (2003). It is generally agreed that providing security, safety and resilience is key for sustainable development of human settlements, e.g., as covered by the United Nations Sustainable Development Goals (United Nations Development Programme 2015).

In summary, there is an increasing need to generate resilient and, hence, sustainable cities with respect to terrorist events. The article addresses this challenge by analysing past terrorism event data to identify critical urban designs on plan level. Weak spots are identified using statistically controlled averaged susceptibilities, vulnerabilities and risks for buildings and infrastructure. This information can be used for discussion and as input for redesign decisions on plan level. Since typically the resource efforts are strongly increasing from concept level to plan level and detail level, decisions on plan level are more efficient.

The article is structured as follows. Section 2 discusses related approaches. Section 3 introduces the present approach. Section 4 details the empirical quantities to assess terroristic threats. Section 5 discusses their statistical quality. Section 6 applies the software-based solution for informed rearrangements of a sample scenario. Section 7 concludes with a detailed outlook as to how the present approach asks for and combines with non-data-driven approaches.

\section{Related Approaches}

General approaches are available for the quantification of terrorism risk. Often, they are based on the quantification of threat and vulnerability in terms of probabilities and conditional probabilities, respectively. They determine the probability of a specified terrorism risk event and consequences in terms of expected damage 
assuming the event occurs (Willis et al. 2005). This approach is taken up in overall semi-quantitative assessments, for instance, as standardized in FEMA (2011) using an event probability, a conditional probability determining the probability that the damage occurs assuming the event occurs and a conditional probability for the expected consequences. The present approach uses historic data to estimate the probability of future terrorist events and their consequences, focusing on attack type and target type combinations relevant for the urban environment.

Woo (2002) derives a quantification of the relative likelihood of targets being selected over severity based on an extended Gutenberg-Richter law as used for seismic risk assessment. It is mainly relevant for insurance finance statistics when compared to the aim of improving urban building and infrastructure designs as the main objective of the present approach.

In the area of hazard and risk analysis for (high) explosive events, the last two decades showed that expert knowledge can be made available in software tools, which support informed users in interactive processes during decision-making. One example of such tools is the Safety Assessment for Explosive Risk (SAFER) (Hardwick et al. 2009). Further examples of developed risk assessment methodologies that were implemented in software tools are the Klotz Group Engineering Tool (van der Voort 2010) and the Explosives Safety Quantitative Risk Analysis (ESQRA) (Salhab et al. 2011) for the risk assessment of ammunition storage and FSQRA for the determination of the safety of moving explosive sources (Häring et al. 2009). Such approaches focus on well-defined application scenarios. In particular, they consider single potential explosive events, or possible events aligned on a three-dimensional line, as in the last example. However, in the present approach, a risk assessment is conducted that takes into account all possible terroristic threats for all buildings and infrastructure in an urban quarter on plan level. In this case, the possible events are distributed on a two-dimensional surface.

The idea of considering multiple possible events for the vulnerability assessment is highly developed for the survivability analysis of technical systems, e.g., when analysing aircrafts with respect to possible impacts (Ball 2003). Such vulnerability modelling approaches are, currently, also extended to urban terrain scenarios, see for instance (Dorsch et al. 2013, 2014). However, their application domain and the military threat types are different when compared to countering terrorism threats in urban planning processes.

\section{Risk Approach to Resilience Enhancement for Urban Environments}

The literature in Sect. 2 shows that quantitative risk assessments for terrorism risks are available, but an application to urban planning applications as the main application scenario has not been performed yet. Initially, within the EU project VITRUV (Fraunhofer 2015), and further within the EU project EDEN (BAE Systems 2015), an interface between available risk assessment methods and urban planning processes has been developed. Aligned with the urban planning process, the approach is applied for different planning stages. 
A typical urban planning process runs through concept, over plan up to the detail level with an increasing level of detail. The paper covers planning phase 2: planning on plan level. It is sandwiched between planning phase 1 conceptual planning, and phase 3 detailed planning. At plan level, the locations of buildings and infrastructure are known without their exact geometry and materials used, i.e., the urban topology is known. The paper details and assesses an approach of how to empower urban planners at plan level to consider and mitigate terrorism risks. This allows to address quarters rather than individual buildings and infrastructure as in standard approaches.

The developed software tool visualizes the results within a three-dimensional graphical user interface. Hence, this solution contributes to the growing change from $2 \mathrm{D}$ to $3 \mathrm{D}$ urban planning processes, as well as their digitalization, as stipulated, for instance, by Building Information Modelling (BIM 2016) and City Geography Markup Language (CityGML 2016) formats. This suits well to business demands of planners, in particular, to visualize their informed planning with respect to intentional threats. It also allows to focus attention at very early planning stages on resilience to terrorism where changes are not yet too expensive.

The present risk-based approach uses the following key definitions:

- Threat: hazard source, type of hazard, attack type, e.g., bombing, arson, chemical threat;

- Building (asset): generalized building, infrastructure type, e.g., bank, apartment house, railway station, company, school, stadium;

- Event: possible terrorist event characterized, i.e., combination of threat and building;

- Susceptibility (frequency, probability): (averaged and/or cumulated) frequencies of events;

- Vulnerability (consequence, damage): (averaged and/or cumulated) damage effects of events on buildings and personnel;

- Risk: (averaged and/or cumulated) local or nonlocal, individual or collective risks;

- Mitigation measures: measures on plan level to mitigate risks.

Obviously, the approach can be used to perform risk management and analysis of urban quarters on plan level with respect to terrorism risks. This becomes obvious when comparing with the five ISO 31000 risk management phases (ISO 2009; Häring 2015): (1) contextualize, (2) identify risks, (3) compute risks, (4) evaluate risks, and (5) mitigate risks. The terrorism risk event identification covers phase (2); the susceptibility, vulnerability and risk quantification covers phase (3); the visualization on urban plan level contributes to phase (4); and phase (5) is covered by the informed possible redesign of scenarios at plan level.

The approach also supports resilience management of terrorism events, which can be resolved in five resilience management phases (Häring 2015; Thoma 2014; Edwards 2009; Thoma et al. 2016; Häring et al. 2016a): (1) prepare, (2) prevent, (3) protect, (4) response, and (5) recover. The preparation phase (1) is supported by the identification of building entities as well as the identification of possible threats. The 
present approach analyses the level of prevention by providing susceptibility quantities, thus contributing to phase (2), and the vulnerability quantities assess the level of protection in case of an event, thus contributing to phase (3). The vulnerability quantities also cover, to some extent, phase (3) because, for instance, the historic number of fatalities in case of an event is also determined by the response phase. The risk quantities can be understood as contributing to phase (1), because they summarize the assessment of prevention, vulnerability and response possible within the present approach, thus allowing for risk-informed mitigation measures the prevention phase (1).

A general formalism how to describe such risk and resilience assessment processes is presented in Häring et al. (2016a), engineering-driven resilience generation processes are presented in Häring et al. (2016b, c). An overview of methods that can be used to populate such processes is given in Häring (2015).

The threats, susceptibilities, vulnerabilities and risks are determined for each building based on historic terrorist event data. For the urban planning process, it is assumed that historic averaged and cumulated data can be used in an informed way to account for future terrorism events. To this end, for instance, historic time windows and worldwide regions or even countries can be selected which are deemed indicative, e.g., for lower and upper bounds of input data. This and further options will be detailed in Sects. 4, 5 and 6.

After this top-level classification of the present approach with respect to risk and resilience management and analysis, the remainder of Sect. 3 will relate key definitions and quantities used in the text to the literature.

The present use of threat is more specified as threat type when compared with often used definitions, see, for instance, the FEMA $(2003,2011)$ definition of threat as "any indication, circumstance, or event with the potential to cause loss of, or damage to an asset". Threat is also not used as synonym for hazard in the sense of a potential event with potential risk, as in the United Nations International Strategy for Disaster Reduction (UN/ISDR 2004)'s definition of hazard as “a potentially damaging physical event, phenomenon or human activity that may cause the loss of life or injury, property damage, social and economic disruption of environmental degradation". Hence, the present approach distinguishes between terroristic threat and terrorist event.

In the present approach, susceptibility (frequency, probability) quantities comprise the cumulated and/or aggregated frequency estimates of future terrorist events. Since these quantities are based on past events, they already include the terrorism threat rate as well as whether terrorism events have been successfully avoided or targets were successfully protected. Susceptibility can be understood as generalization of the probability component of the classical risk definition, e.g., formulated by Smith (2013) to be "the combination of the probability of a hazardous event and its negative consequence". This is different from the definition of Brinkmann (2007), where susceptibility encompasses the weakness of an exposed system or element to a threat (and is part of an extensive vulnerability concept). In the field of aircraft survivability, the term susceptibility is defined as inability to avoid a threat (Ball 2003), which is strongly linked to the frequency of threat events 
as in the present definition. The present approach also links with the definition of exposure to threat as defined by Morgan and Henrion (1992).

In a similar way, vulnerability [(what-if) consequence, damage] is defined as a cumulated and/or aggregated empirical consequence measure of historic terrorist events. This is a restricted definition when compared to often much broader definitions or usage as in sociology (Branscomb 2006), urban and terrorism sociology (Coaffee 2008, 2009), human geography (Adger 2006), climate change research (Füssel 2007), or sustainability science (Turner et al. 2003). However, since it rests on the evaluation of historic terrorism events, it implicitly takes into account many of the factors (aspects) of vulnerability accounted for in conceptual and analytical frameworks, e.g., organizational measures that reduce the damage in case of events. The present definition is a generalization of the classical measure for consequence (damage) in the classical definition of risk. It is suitable for the present data-driven statistical-engineering approach which aims at quantitative vulnerability and risk analyses. It contributes to analyses of components of critical infrastructure rather than overall critical infrastructure systems as described by Kröger and Zio (2011), which takes account of the interconnectedness and dependence of components. The present approach focuses on consequences for humans. Building (asset) values lost in case of events are further options (FEMA 2003, 2011).

Finally, various averaged and/or cumulated risk quantities are derived from terrorist event data. Here, it is assumed that the single risks are both proportional to the defined susceptibility (frequency, probability) and vulnerability (damage, consequence) quantities as in the classical definition of risk $R=F \times C$ (Kaplan and Garrick 1981). This also corresponds to best practice engineering approaches, see e.g., (Ale 2002). Risk aversion factors are not taken into account, because the present approach aims to contribute to risk management phase (3) rather than (4), as discussed above. However, factors are allowed for that can be used to modify the empirical susceptibility, vulnerability and risk quantities if additional urban planning information is available.

In summary, the concepts used in the present approach for terrorism susceptibility, vulnerability and risk quantification based on empirical event data take up current concepts and notions as far as deemed relevant for the application domain, urban planning on plan level, with the aim of countering effects of terrorism in urban areas. In Sect. 4, the approach is quantified. Section 5 shows how to guarantee statistical accuracy, and Sect. 6 shows how to use the quantities to identify weak spots in the urban design, being aware of statistical uncertainties and deep uncertainties.

\section{Empirical Susceptibility, Vulnerability and Risk Analysis}

\subsection{Overview}

In the following, risk is interpreted as generalization of the product of quantities relating to the frequency and to the consequences of a hazardous event. If urban structures and buildings are exposed to a threat, for the determination of the 


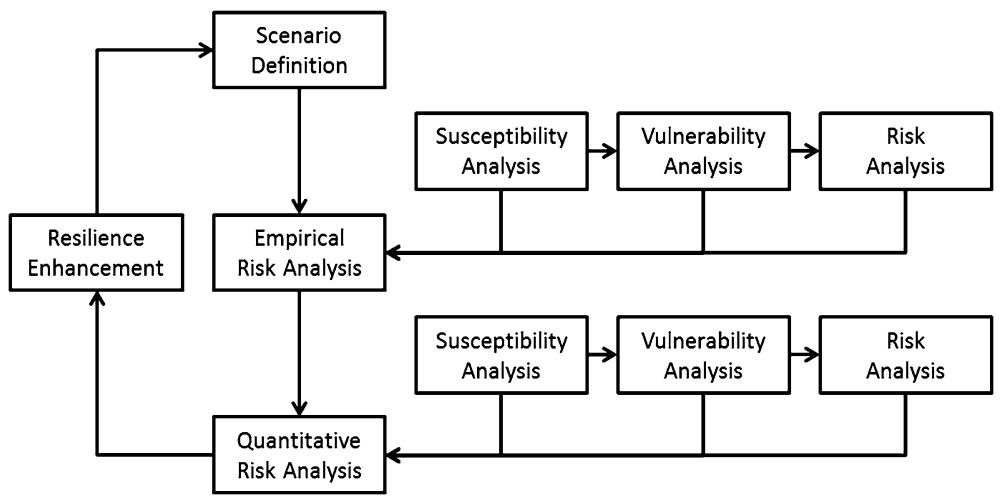

Fig. 1 Scheme for empirical and quantitative susceptibility, vulnerability and risk analysis to improve the resilience of urban areas

frequency and consequence quantities, the terms susceptibility and vulnerability analysis are used as motivated in Sects. 2 and 3, see Fig. 1. After the definition of a scenario, comprising typically the urban design and the selection of terroristic threats, two different risk analysis schemes can be applied to generate generalized risk quantities: empirical and quantitative analyses.

The empirical analysis uses historical data to quantify the risk for urban objects and is a broad and fast risk analysis scheme. The quantitative risk assessment procedure uses in addition educated assumptions about the local distribution of attack events to refine the susceptibility analysis as well physical-engineering and simulative models for the quantification of local and global, individual and collective risk quantities. In this sense, of course both approaches are quantitative. The paper describes in detail the empirical risk assessment approach.

In both cases, the consideration and selection of resilience enhancement measures follow after the discussion and evaluation of analysis results. Of course, when focusing on the plan level only, the more refined quantitative analysis can be omitted.

The present journal paper contains the first description of the quantitative empirical approach as well as of the statistical accuracy challenges. The current quantitative engineering-simulative approach is described in Fischer et al. (2016). A first description of the overall approach consisting of the empirical and quantitative assessment of terrorist events for urban areas is given by Voss et al. (2012) and details most of the empirical and analytical-engineering-simulative concepts and formal quantities to be used and implemented in the course of the VITRUV and EDEN projects, respectively. In particular, it details how to use multiple weighted events and local event densities between buildings to compute averaged susceptibilities, vulnerabilities and risks, as further advanced in Sects. 2 and 3.

The empirical-historical terrorism event data basis of the empirical and quantitative analyses, the Terror Event Database (TED) has first been described in Siebold et al. (2009). Besides a further detailed analysis of the terrorism event data, Fischer et al. (2014) also give examples of mapping selected empirical 
assessment quantities on urban environments. They describe the ontology used within the database as well as how to identify worldwide regions of different level of terroristic activity.

An overview on the intermediate and final overall implemented approach including sample applications as achieved within the VITRUV project and at the beginning of the EDEN project is given by Fischer et al. (2012) and Riedel et al. (2014), respectively. The latter article shows that also consequences in terms of progressive collapse of buildings and traffic congestions can be handled. It documents the usage of simulation tools for the hazard propagation modelling of chemical agents as well as the corresponding local damage assessment. It also indicates that the approach can be extended to natural hazards. In Riedel et al. (2015), the quantitative approach is sketched with a strong focus on the comparison of protected and unprotected scenarios and an efficiency assessment of physical counter-measures.

Figure 2 shows a detailed urban plan assessment based on the empirical risk analysis. This approach links with standardized approaches for risk management, in particular, ISO 31000 (ISO 2009). The first step includes the model generation of the urban area. For the definition of urban objects, physical and qualitative parameters are used. Next, the geographic risk category is selected. The analysis of historical data shows significant differences concerning frequencies and consequences depending on the region, see (Fischer et al. 2014) and Figs. 7, 8 and 9 of the "Appendix". For instance, the state of (the expected future) development of a region is a criterion for the selection of empirical data for the empirical risk assessment.

The definition of the generalized building type (see Fig. 2) is based on a detailed analysis of the empirical data, taking account of the usage of the buildings (see also Fig. 3). Scaling factors can be used to consider physical, societal or economical properties of the considered urban area that allow to argue that the statistical historical estimates have to be modified. The calculation of the empirical averaged risk includes, with the computation of the susceptibilities and vulnerabilities, all steps of a risk assessment approach, in particular, generalizations of probability and consequence analysis. The last step of Fig. 2 is a risk management process step. If

Fig. 2 Exemplary steps to perform an empirical risk analysis for urban areas. In a similar way, susceptibility and vulnerability quantities can be assessed

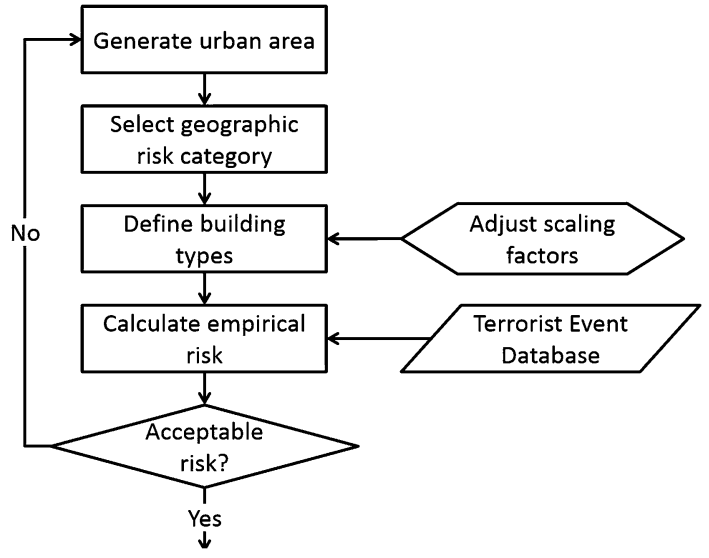




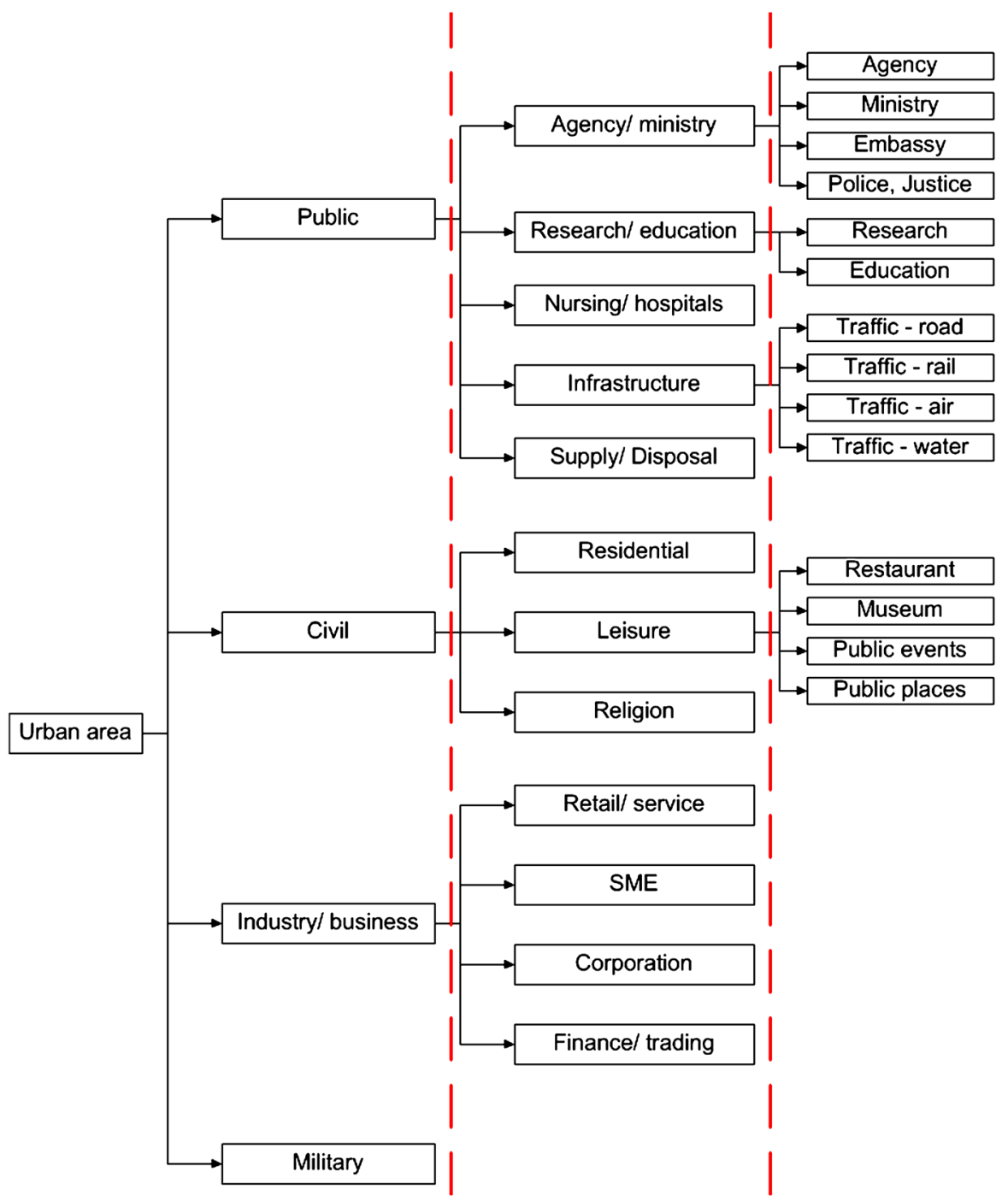

Fig. 3 Arborescence of an urban environment in dependency of the building usage

the calculated averaged risks (or susceptibilities, vulnerabilities) are not acceptable, risk minimization measures must be applied before starting a further assessment process.

The approach uses the information which is known on an early stage of planning (Plan Level). At this level, it is assumed not to know much more details than region, threat type and usage of the buildings. So, the averaging is done on this level. In the project VITRUV, it was discussed and decided with urban planners that this information in the phase of urban planning is available and sufficient. 
Next, Sects. 4.2, 4.3, 4.4 and 4.5 introduce all quantities of the empiricalhistorical statistical approach. Section 5 describes the data sources used and assesses the statistical accuracy of the quantities.

\subsection{Basic Definitions: Region, Threat, Building, Area and Scaling Factors}

Geographic risk categories are distinguished (Fischer et al. 2014). Let $G_{h}, h \in$ $\left\{1, \ldots, n_{\text {geographiccategory }}\right\}$, be the $n_{\text {geographiccategory }}$ distinct geographic regions. Let $T_{i}^{\text {threat }}, \quad i \in\left\{1, \ldots, n_{\text {threat }}\right\}$, be threat types. The urban environment is given by a set of buildings or structures with position vectors $\vec{b}_{k}, k \in\left\{1, \ldots, n_{\text {building }}\right\}$, and building types $T_{l}^{\text {building }}, l \in\left\{1, \ldots, n_{\text {building type }}\right\}$, e.g., governmental, private building or embassy.

Multiplicative weighting factors are considered for the frequency of hazardous events as well as for the consequences. In general, there exist distinct geometric areas $A_{m}, m \in\left\{1, \ldots, n_{\text {geometricareas }}\right\}$, in the urban environment, e.g., defined as plane polygons and corresponding to parts of urban quarters, parts or sets of streets, where the same multiplicative weighting factors (influencing factors) $w_{m}^{i k}$ (for the frequency) and $v_{m}^{i k}$ (for the consequences) can be defined which are valid for all buildings on this areas. Also, they are also defined to depend on a combination of building and threat and result in overall weights for each combination of building and threat:

$$
w_{i k}=\prod_{m=1}^{n_{i k}^{\text {weight }}} w_{m}^{i k} \quad \text { and } \quad v_{i k}=\prod_{m=1}^{n_{i k}^{\text {weight }}} v_{m}^{i k} .
$$

These weighting factors for urban subareas can be used to describe social, environmental, economic or other security-relevant influences that may increase or decrease the likelihood of the occurrence of a threat event $T_{i}^{\text {threat }}$ in the neighbourhood of or within a building $\vec{b}_{k}$ as well as the measures of its consequences.

Examples for influences on frequency include the lack of social cohesion or the "broken windows effect" (Kelling and Wilson 1982). Examples for effects on consequences are personnel protective equipment, fast emergency measures, reduced response time, and physical counter-measures. Examples for physical measures include, e.g., structural retrofit of masonry walls (Riedel et al. 2012), reinforced concrete walls (Stolz et al. 2014), reduction of window sizes, blast walls (Smith 2010), blast curtains, blast-resistant glazing and membrane structures (Gebbeken and Döge 2010). For instance, by assuming that minimum distances can be guaranteed, fast distribution-based assessments could be used to estimate the scaling factors on consequences, for instance, by either using, directly, geometry data as input (Häring and Gürke 2006) or indirectly first estimating the loading and, then, the damage effects (Aschmoneit et al. 2009; Aschmoneit and Häring 2010).

Equation (1) can be summarized as the risk reduction or enhancement quantification due to societal effects and physical effects on event frequencies 
and, to a lesser extent, also on consequences relative to the respective historical average data. If such factors are used, whether increasing or decreasing, they must be credibly justifiable at plan level, e.g., based on past detailed assessment of similar scenarios or estimated within expert rounds. In the application example of Sect. 5, all these factors are set to unity. It is beyond the scope of the article to discuss the determination of the values of the factors further.

All consequences for persons and infrastructure arising from the threats $T_{i}^{\text {threat }}$ are separated into different types of consequences (damage types) $T_{n}^{\text {consequence }}, \quad n \in$ $\left\{1, \ldots, n_{\text {consequence }}\right\}$, e.g., injuries or structural building damage. This definition allows the comparison of effects of different types of events in terms of the same consequences.

\subsection{Empirical Frequency and Susceptibility}

As presented in Sect. 3, risk $R$ is classically defined as the product of a frequency measure $F$ of an event and a consequence measure $C$ in case the event happens. To include the influencing factors of Eq. (1), this is taken up in the following top-level definition of risk as motivated in Sect. 3:

$$
R=F w C v .
$$

The empirical approach determines the event frequency with empirical data from the time interval $\left[t_{1}, t_{2}\right]$. For a specific combination of threat type $T_{i}^{\text {threat }}$ and building type $T_{l}^{\text {building }}$, the average absolute event frequency is given for each geographic region by:

$$
\begin{aligned}
& F\left(T_{l}^{\text {building }}, T_{i}^{\text {threat }},\left[t_{1}, t_{2}\right], G_{\mathrm{h}}\right. \\
& )=\frac{\text { number of events of type } T_{i}^{\text {threat }} \text { on building type } T_{l}^{\text {building }} \text { within }\left[t_{1}, t_{2}\right] \text { in region } G_{h}}{\text { length of time period } \times \text { number of buildings of type } T_{l}^{\text {building }} \text { in region } G_{h}} \\
& =\frac{N^{\text {events }}\left(T_{i}^{\text {threat }}, T_{l}^{\text {building }},\left[t_{1}, t_{2}\right], G_{h}\right)}{\left(t_{2}-t_{1}\right) N_{l}^{\text {building }}\left(G_{h}\right)} .
\end{aligned}
$$

The indices $h, i$ and $l$ are restricted as defined in Sect. 4.2. Typical raw data used for the computation of (3) are given in Figs. 7, 8 and 9 in the "Appendix". Incorporating the weighting factors $w_{i k}$ for a specific urban region or even for a single building, the susceptibility of a specific building at $\vec{b}_{k}$ to a specific threat $T_{i}^{\text {threat }}$ in region $G_{h}$ is defined as:

$$
\begin{aligned}
S\left(\vec{b}_{k}, T_{i}^{\text {threat }},\left[t_{1}, t_{2}\right], G_{h}\right) & =S\left(T^{\text {building }}\left(\vec{b}_{k}\right), T_{i}^{\text {threat }},\left[t_{1}, t_{2}\right], G_{h}\right) \\
& =F\left(T^{\text {building }}\left(\vec{b}_{k}\right), T_{i}^{\text {threat }},\left[t_{1}, t_{2}\right], G_{h}\right) w_{i k} .
\end{aligned}
$$

Here, the time interval in Eq. (4) tells that the data used for the estimation of the frequency of (3) and (4) are obtained using data out of this time interval, e.g., the few most recent years. 
By summing over the threats $T_{i}^{\text {threat }}$, the susceptibility $S$ of a specific building $\vec{b}_{k}$ to all threats $T_{i}^{\text {threat }}$ is given by:

$$
S\left(\vec{b}_{k},\left[t_{1}, t_{2}\right], G_{h}\right)=\sum_{i=1}^{n_{\text {threat }}} F\left(T^{\text {building }}\left(\vec{b}_{k}\right), T_{i}^{\text {threat }},\left[t_{1}, t_{2}\right], G_{h}\right) w_{i k},
$$

resulting in a threat-cumulated susceptibility, a cumulated frequency.

In a similar way, it can be summed over a set of buildings, e.g., all embassies of a town, arriving at a cumulated susceptibility for a given building type of an urban region.

\subsection{Empirical Consequences and Vulnerability}

In the present paper, the calculated vulnerability is based on the consequences of past events caused by different threats $T_{i}^{\text {threat }}$. The consequences are defined for each combination of consequence type, threat type, building type and geographic region. It is given as the average total number of consequences, e.g., the total number of casualties, over the number of events of the chosen combination. Again, empirical data of a certain time period are used.

The average empirical consequences per event and building reads in summarizing notation:

$$
\begin{aligned}
& C\left(T_{i}^{\text {threat }}, T_{l}^{\text {building }}, T_{n}^{\text {consequence }},\left[t_{1}, t_{2}\right], G_{h}\right) \\
& =\frac{\text { number of } T_{n}^{\text {consequence }} \text { for } T_{l}^{\text {building }} \text { and } T_{i}^{\text {threat }} \text { within }\left[t_{1}, t_{2}\right] \text { in region } G_{h}}{\text { number of } T_{i}^{\text {threat }} \text { events on } T_{l}^{\text {building }} \text { within }\left[t_{1}, t_{2}\right] \text { in region } G_{h}} \\
& =\frac{N^{\text {consequences }}\left(T_{i}^{\text {threat }}, T_{l}^{\text {building }},\left[t_{1}, t_{2}\right], G_{h}\right)}{N^{\text {events }}\left(T_{i}^{\text {threat }}, T_{l}^{\text {building }},\left[t_{1}, t_{2}\right], G_{h}\right)} .
\end{aligned}
$$

The damage quantity (6) is the average damage of type $T_{n}^{\text {consequence }}$ per threat event of type $T_{i}^{\text {threat }}$ targeting on building type $T_{l}^{\text {building }}$ in a region $G_{h}$.

Based on this average damage per event and incorporating the weighting factors $v_{i k}$ for a specific urban region or even for a single building, to a specific threat $T_{i}^{\text {threat }}$ in region $G_{h}$, the vulnerability of a specific building at $\vec{b}_{k}$ to a specific threat $T_{i}^{\text {threat }}$ in region $G_{h}$ is defined as:

$$
\begin{aligned}
& V\left(T_{i}^{\text {threat }}, T_{l}^{\text {building }}, T_{n}^{\text {consequence }},\left[t_{1}, t_{2}\right], G_{h}\right) \\
& \quad=C\left(T_{i}^{\text {threat }}, T_{l}^{\text {building }}, T_{n}^{\text {consequence }},\left[t_{1}, t_{2}\right], G_{h}\right) v_{i k} .
\end{aligned}
$$

By averaging over all threat types $T_{i}^{\text {threat }}$, the homogeneous average vulnerability of a building of type $T_{l}^{\text {building }}$ with respect to consequence type $T_{n}^{\text {consequence }}$ for all threat types is calculated as: 
$V\left(T_{l}^{\text {building }}, T_{n}^{\text {consequence }},\left[t_{1}, t_{2}\right], G_{h}\right)=\frac{1}{n_{\text {threat }}} \sum_{i=1}^{n_{\text {threat }}} C\left(T_{i}^{\text {threat }}, T_{l}^{\text {building }}, T_{n}^{\text {consequence }},\left[t_{1}, t_{2}\right], G_{h}\right) v_{i k}$.

In a very similar way, a certain subset of threats can also be used for averaging, e.g., all kinds of explosive threats.

Similar to Eq. (8), vulnerabilities averaged over building types can also be computed by summing over a subset of building types and taking into account the number of building types in the subset as pre-factor as in (8) the number of threats. This generates a homogeneous average vulnerability with respect to a building type subset for a given threat.

It can also be summed and averaged over a subset of threats and a subset of building types simultaneously. This generates a homogeneous average vulnerability with respect to (a set of) building types and (a set of) threats.

Obviously, it can only be summed over a subset of consequence types, if all consequences are expressed in the same units, which is often not feasible or even desirable, e.g., when comparing human damage and building damage.

It has to be mentioned that Eq. (8) must be used carefully, because categorization into $T_{i}^{\text {threat }}$ is an empirical setting, and the respective averaging is conducted without considering the number of events per category. As a result, different categorizations can lead to different averaged homogeneous vulnerabilities using the same data. However, this can be overcome using well-defined threat categories representing a similar number of threat events, by generating (8) with an expression similar to Eq. (6) ab initio, or finally by guaranteeing that the basic empirical input data of (6) are sufficiently accurate. The present approach follows, in Sect. 5, the latter option.

In Eqs. (7) and (8), the vulnerabilities are defined as averaged damage (consequence) which are further homogeneously averaged with respect to threat types in the case of (8) and the discussed similar expressions. As described, it can also be homogenously averaged twice, e.g., by averaging over a set of building types and a set of consequences.

\subsection{Empirical (Averaged) Risk}

To obtain the empirical absolute risk of a single building $\vec{b}_{k}$ for threat $T_{i}^{\text {threat }}$ and

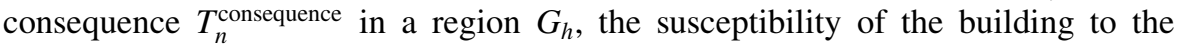
threat and the damage caused by the threat are multiplied:

$$
\begin{aligned}
& R\left(\vec{b}_{k}, T_{i}^{\text {threat }}, T_{n}^{\text {consequence }},\left[t_{1}, t_{2}\right], G_{h}\right) \\
& =S\left(T^{\text {building }}\left(\vec{b}_{k}\right), T_{i}^{\text {threat }},\left[t_{1}, t_{2}\right], G_{h}\right) V\left(T_{i}^{\text {threat }}, T^{\text {building }}\left(\vec{b}_{k}\right), T_{n}^{\text {consequence }},\left[t_{1}, t_{2}\right], G_{h}\right) \\
& =F\left(T^{\text {building }}\left(\vec{b}_{k}\right), T_{i}^{\text {threat }},\left[t_{1}, t_{2}\right], G_{h}\right) w_{i k} C\left(T_{i}^{\text {threat }}, T^{\text {building }}\left(\vec{b}_{k}\right), T_{n}^{\text {consequence }},\left[t_{1}, t_{2}\right], G_{h}\right) v_{i k} .
\end{aligned}
$$


By summing over all threats $T_{i}^{\text {threat }}$, the risk for a building $\vec{b}_{k}$ and a consequence type $T_{n}^{\text {consequence }}$ in a region reads:

$$
\begin{aligned}
& R\left(\vec{b}_{k}, T_{n}^{\text {consequence }},\left[t_{1}, t_{2}\right], G_{h}\right) \\
& =\sum_{i=1}^{n_{\text {threat }}} F\left(T^{\text {building }}\left(\vec{b}_{k}\right), T_{i}^{\text {threat }},\left[t_{1}, t_{2}\right], G_{h}\right) w_{i k} C\left(T_{i}^{\text {threat }}, T^{\text {building }}\left(\vec{b}_{k}\right), T_{n}^{\text {consequence }},\left[t_{1}, t_{2}\right], G_{h}\right) v_{i k} .
\end{aligned}
$$

The total risk of all buildings in the urban scenario in a specific region with respect to a consequence type is obtained by summing over all buildings $\vec{b}_{k}$ :

$$
R\left(T_{\mathrm{n}}^{\text {consequence }},\left[t_{1}, t_{2}\right], G_{h}\right)=\sum_{k=1}^{n_{\text {building }}} R\left(\vec{b}_{k}, T_{n}^{\text {consequence }},\left[t_{1}, t_{2}\right], G_{h}\right) .
$$

For a given time interval $t_{3}, t_{4}$, the expected total damage numbers (e.g., total number of injured) in the scenario can be calculated by multiplying the total risk of buildings for a specific consequence type $T_{n}^{\text {consequence }}$ with the given length of the time period (interval) considered:

$$
N\left(T_{n}^{\text {consequence }},\left[t_{3}, t_{4}\right], G_{h}\right)=R\left(T_{n}^{\text {consequence }},\left[t_{1}, t_{2}\right], G_{h}\right)\left(t_{4}-t_{3}\right) .
$$

Similar to Eqs. (11) and (12), the risk and the total risk number expressions can also be derived at building level, sets of buildings as well as for groups of types of threats only. However, again, only if a single measure of consequences for all types of damage is available, e.g., monetary loss, it can be summed over all consequence types.

As can be observed from Eqs. (9)-(12), the risk quantities take into account the susceptibilities (empirical frequencies) of the threats. In particular, Eq. (10) can be understood as an average vulnerability taking also empirical event frequency data into account, i.e., going beyond the homogeneous threat type averaging of Eq. (8) and the discussed similar expressions. Therefore, only the risk quantities use all empirical information for assessment. This advantageous and standard option of combination of empirical analyses originates from the fact that event frequencies can be considered without distinguishing between consequence types. However, damage effects depend on building and threat types as well as the selected damage type. Nevertheless, also in the latter cases, averaging is feasible, the most meaningful average being the defined risk quantities.

\section{Data Source Description, Practical Implementation and Statistical Accuracy}

Section 5.1 gives an overview of the empirical hierarchical data set and shows how to use more data in case the accuracy does not suffice. Sections 5.2, 5.3 and 5.4 show how to determine the statistical accuracies for the susceptibility (frequency), vulnerability (consequence) and risk quantities, respectively. 
The statistical accuracy assessment uses the basic expressions for susceptibility (3), vulnerability (6) and risk (9), because on plan level, the finest reasonable resolution is to look at a single building or infrastructure. Therefore, it has to be decided for which combinations of building and threat type and respective generalizations, sufficient data sets are available to determine these quantities with sufficient accuracy.

Sections 5.2 and 5.3 use confidence intervals defined by multiplicative factors (rather than by absolute values). They show how to achieve given intervals for given accuracies for the hierarchical data set. This allows order-of-magnitude assessments of averaged susceptibilities, vulnerabilities and risks as appropriate for plan-level urban terrorism assessment and as applied in the sample application case of Sect. 6.

\subsection{Database and Empirical Input Data Used}

The empirical risk analysis is based on historical data extracted from the Terror Event Database (TED) at Fraunhofer EMI (Siebold et al. 2009). Sample applications and, in particular, topic-specific extensions as well as differences of TED to existing databases are also detailed in (Siebold et al. 2009) focusing on statistical methods, (Ziehm et al. 2009) focusing on terrorist events on airports and (Ziehm and Häring 2011) on terrorist events in harbours. The geographic regions $G_{h}$ used are motivated and defined in Fischer et al. (2014). Figures 7, 8 and 9 in the "Appendix" give an overview of the data used.

Inspecting Eqs. (3) and (6), the empirical data are available in the form of numbers of events over number of buildings, $N^{\text {events }} / N^{\text {building }}$, and numbers encoding consequences (e.g., number of casualties) over number of events, $N^{\text {consequences }} / N^{\text {events }}$. Hence, the accuracy of statistical values for frequency and consequences depends on the values for $N^{\text {events }}, N^{\text {building }}$ and $N^{\text {consequences }}$.

To express how advantage is taken of the different levels of resolution of the database, graph theory is used. This allows to increase or decrease the data quantities according to the statistical data available and according to statistical confidence requirements. The graph theory for arborescence is selected. An arborescence is a directed, rooted tree in which all edges point away from the root. Using these insights, a hierarchical definition of an urban area is defined in dependency of the usage of buildings and infrastructures it is composed of, see Fig. 3. It can also be understood as an ontology of the generalized building types. Similar models can be defined for the threat types and the geographical regions.

If empirical data are very poor for a building type, the data of a parent vertex can be used instead of the current vertex (e.g., increasing $N^{\text {events }}$ and $N^{\text {building }}$ ). Vice versa, a child vertex can be applied to get further refinement, if the statistical accuracy suffices. For example, "Agency/ministry" in Fig. 3 could be refined to the children "Agency", "Ministry", "Embassy" or "Police, Justice" if there are still enough data, respectively, or it could be generalized to the parent "Public" if more data are needed.

With the described hierarchical model, it becomes obvious that the quantity of input data for Eqs. (3) and (6) can be increased or decreased in meaningful steps controlled by the arborescence. For instance, "Agency/Ministry" is a meaningful generalized building type for all its children and can be used for urban planning. 
For the building-, region- and threat-type-dependent empirical data, the statistical accuracy has to be determined. For a requested accuracy, the minimum input raw data are calculated for the empirical frequency and consequences in Sects. 5.2 and 5.3. The accuracy of the risk quantities is derived using error propagation in Sect. 5.4. The results documented in Clauset et al. (2007) are used for comparison of the developed distribution function for the consequences in Sect. 5.3.

\subsection{Accuracy of Susceptibility Quantities}

First, the susceptibility (frequency) is discussed. Based on the data which are available as described in Sect. 5.1, the multiplicative error bar for the frequency is computed.

The accuracy analysis is based on the following assumptions:

- The number of buildings of a building type $T_{l}^{\text {building }}$ in a specific region $G_{h}$ is known without error.

- The probability of an attack for each building of a specific building type is independent and identically distributed for each region $G_{h}$.

Therefore, the binominal distribution can be used to determine the statistical accuracy of the frequencies of Eqs. (3), (4) and (5).

The second assumption is used because of the following reasons. It is a typical first statistical choice and benchmark. In the present case, the paper shows that even with these assumptions, it is challenging to generate results with sufficient statistical accuracy. It even turns out to be necessary to increase the input data sets in a systematic way. Therefore, for more advanced approaches, the data will not suffice for covering the wide range of possible terrorist events.

Further arguments for the present choice include: Terrorist events are rare events separated by space, time, and, to some degree, also attack type and attack means. This hints at their independence. The approach used distinguishes with respect to combinations of attack type, building usage and geographic region. Within such a cluster to use the same distribution is a less-restrictive assumption or at least a more accessible assumption, which can be challenged during the evaluation of assessments. For instance, if an area is less and less accessible by trucks, the corresponding event frequency can be changed. Furthermore, when considering longer periods in time, the historical event data already take account of the ever ongoing cat-and-mouse game of the ongoing 'terror innovations' in an averaging way.

In statistics, a confidence interval is an interval estimate of a population parameter and is used to determine its reliability. The Clopper-Pearson interval (Clopper and Pearson 1934) is an early and common method for calculating binomial confidence intervals. It is used to calculate the error bar for the frequency if there are $x$ events (corresponding to $N^{\text {events }}$ of a selected building and attack type combination) on $n$ buildings (corresponding to $N^{\text {building }}$ ). In this case, the empirical frequency estimate reads $\bar{p}=x / n$. 
The following paragraphs first show how to determine an interval $\left[p_{\min }, p_{\max }\right]$ in which the real value $p$ lies with a proportion of more than $1-\alpha$. The main goal is to determine, for a given multiplicative accuracy $a_{\text {Susceptibility }}$ for the susceptibility (3) and error level $\alpha$, the minimum number of $x_{\min }\left(a_{\text {Susceptibility }}\right)$ of sufficient event data such that the multiplicative confidence assessment (15) holds.

The real values $p$, which are within the requested error level $\alpha$, are represented as:

$$
\left\{p \mid P[\operatorname{Bin}(n ; p) \leq x] \geq \frac{\alpha}{2}\right\} \cap\left\{p \mid P[\operatorname{Bin}(n ; p) \geq x] \geq \frac{\alpha}{2}\right\},
$$

where $x$ corresponds to the number of successes observed in the sample and $\operatorname{Bin}(n, p)$ is the binomial random variable with $n$ trials and probability of success $p$.

Because of the relationship between the cumulative binomial distribution and the beta distribution $B$, the Clopper-Pearson interval reads:

$$
p_{\min }=B\left(\frac{\alpha}{2} ; x, n-x+1\right)<p<B\left(1-\frac{\alpha}{2} ; x+1, n-x\right)=p_{\max } .
$$

Table 1 shows the relative confidence interval for exemplary values. Only if the number of buildings is large, there is hardly any difference to the Poisson distribution ( see $n=\infty$ ).

For the considered cases, besides the error bars, for a practical application on the arborescence as described in Sect. 5.1, the minimum number of events $x_{\min }$ (corresponding to $N^{\text {events }}$ ), given the desired multiplicative accuracy $a_{\text {Susceptibility }}$ and the desired error level $\alpha$, must be determined. The accuracy is defined to be multiplicative, because for the analyses, it is advantageous to obtain correct orderof-magnitude estimates of the susceptibility and vulnerability, i.e., the correct frequency is a factor greater or smaller than the empirical frequency $\bar{p}$. Hence, $x_{\text {min }}\left(a_{\text {Susceptibility }}\right)$ is determined so that the multiplicative confidence interval,

$$
\bar{p} / a_{\text {Susceptibility }}<p<a_{\text {Susceptibility }} \bar{p},
$$

Table 1 Confidence intervals $\left[p_{\min }, p_{\max }\right]$ in units of $\bar{p}=x / n$ for $x$ events for $n$ buildings and an error level of $\alpha=5 \%$

\begin{tabular}{lllll}
\hline$x$ & $n$ & $\bar{p}=x / n$ & $p_{\min } / \bar{p}$ & $p_{\max } / \bar{p}$ \\
\hline 5 & 10 & 0.5 & 0.37417 & 1.62583 \\
5 & 100 & 0.05 & 0.32864 & 2.25670 \\
5 & 1000 & 0.005 & 0.32508 & 2.32589 \\
5 & $1,000,000$ & 0.000005 & 0.32470 & 2.33366 \\
5 & $\infty$ & & 0.32470 & 2.33367 \\
10 & 100 & 0.1 & 0.49005 & 1.76223 \\
10 & 1000 & 0.01 & 0.48055 & 1.83132 \\
10 & $1,000,000$ & 0.00001 & 0.47954 & 1.83903 \\
10 & $\infty$ & & 0.47954 & 1.83903 \\
100 & 1000 & 0.1 & 0.82105 & 1.20288 \\
100 & $1,000,000$ & 0.0001 & 0.81365 & 1.21625 \\
100 & $\infty$ & & 0.81364 & 1.21627 \\
\hline
\end{tabular}


is valid with a proportion of more than $1-\alpha$. How is $x_{\min }$ obtained? For $x$ fixed and, respectively, fixed values of $a_{\text {Susceptibility }}, n$ and $\alpha$, the confidence interval $\left[p_{\min }, p_{\text {max }}\right]$ is computed according to Eq. (14). It can be observed from Table 1 that increasing $x$ while fixing $n$ decreases the confidence interval. To obtain $x_{\min }$, the value of $x$ is increased in integer steps until the confidence interval is just within the multiplicative boundaries of (15).

Exemplary results can be found in Table 2. For instance, 7 events suffice for a multiplicative accuracy of 2 as defined in (15) for the susceptibility of events in case of 100 buildings.

In the case of large numbers of buildings $N^{\text {building }}$ and small probabilities $p$, Poisson distribution can be used instead of the binominal distribution, and hence, the number of buildings is not relevant for determining the accuracy $a_{\text {Susceptibility. }}$ In this case, only the Poisson distribution parameter $\lambda$ must be determined so that,

$$
\sum_{k=0}^{\lambda} \frac{e^{-\lambda / a}(\lambda / a)^{k}-e^{-\lambda a}(\lambda a)^{k}}{k !} \geq 1-\alpha .
$$

This is used for the last column of Table 2. In statistics, it is approved to use this approach if the probability $p<0.01$. Hence, the easier approach (16) can be used for the computations at the right-hand side of the red line of Table 2 instead of the Binomial approach, see in particular, the last two columns of Table 2. Another interesting result of Table 2 is that, if only a multiplicative accuracy $a_{\text {Susceptibility }} \geq 5.3$ is needed, one event is enough to achieve an error level not exceeding $5 \%$. This accuracy suffices to perform order-of-magnitude estimates.

Figure 4 shows the narrowing of the confidence bands for the empirical susceptibility for an increasing number of events. It depicts the upper and lower bound for the number of events that result in frequencies that lay within the confidence interval $\left[p_{\min }, p_{\max }\right]$ according to (14) (see the yellow vertical lines) in dependency of the empirical number of events. For a greater number of events also,

Table 2 Minimum number of events to obtain an error level $\alpha \leq 5 \%$ in dependency of the number of buildings $N^{\text {building }}$ and the multiplicative accuracy $a_{\text {Susceptibility }}$

\begin{tabular}{|r|r|r|r|r|r|r|r|}
\hline$a_{\text {Susceptibility }} \backslash N^{\text {building }}$ & $\mathbf{1 0 0}$ & $\mathbf{5 0 0}$ & $\mathbf{1 0 0 0}$ & $\mathbf{1 0 0 0 0}$ & $\mathbf{1 0 0 0 0 0}$ & $\mathbf{1 0 0 0 0 0 0 0}$ & $\infty$ \\
\hline $\mathbf{1 , 0 5}$ & 94 & 382 & 617 & 1389 & 1588 & 1611 & 1614 \\
\hline $\mathbf{1 , 1}$ & 81 & 229 & 297 & 406 & 421 & 423 & 423 \\
\hline $\mathbf{1 , 2}$ & 53 & 94 & 104 & 114 & 115 & 116 & 116 \\
\hline $\mathbf{1 , 5}$ & 19 & 22 & 23 & 23 & 23 & 23 & 23 \\
\hline $\mathbf{2}$ & 7 & 8 & 8 & 8 & 8 & 8 & 8 \\
\hline $\mathbf{3}$ & 3 & 3 & 3 & 3 & 3 & 3 & 3 \\
\hline $\mathbf{5}$ & 2 & 2 & 2 & 2 & 2 & 2 & 2 \\
\hline$\geq \mathbf{5 , 3}$ & 1 & 1 & 1 & 1 & 1 & 1 & 1 \\
\hline
\end{tabular}


the multiplicative accuracy is decreasing; see the exemplary red vertical lines. A further discussion of some of the shown building types is given in Sect. 6 .

In Fig. 4, the binomial distribution is indistinguishable from the Poisson distribution for the range shown. The number of buildings is already $10^{5}$ for "Nursing, hospital". See Table 2 for the comparison of the distributions. The examples are given for third- and fourth-column vertexes of building usage, see Fig. 3.

In summary, the expected minimum number of events $x_{\min }$ in the database for a given accuracy factor $a_{\text {Susceptibility }}$, proportion $1-\alpha$ and number of buildings $N^{\text {building }}$ is calculated. If the needed number $x_{\min }$ is not given, the parent vertex is used as described in Sect. 5.1.

For application in the VITRUV project, it was decided in consultation with endusers to use a multiplicative accuracy of $a_{\text {Susceptibility }}=2$. In this case with (at least) $95 \%$ probability, the correct values lie within the interval defined in (15). If the database holds less than eight events, the tool automatically goes to the next parent vertex unless there are at least eight events. For the case of application, the number of most buildings $N^{\text {building }}$ is in the range of $10^{5}$ up to the order of $10^{8}$. Only in special cases like embassies, low orders of about $10^{3}$ are reached. Only in such cases, the binomial distribution must be used to determine $x_{\min }$. The values of $N^{\text {events }}$ are typically in the order of 2-3000, see also Fig. 7 of the "Appendix".

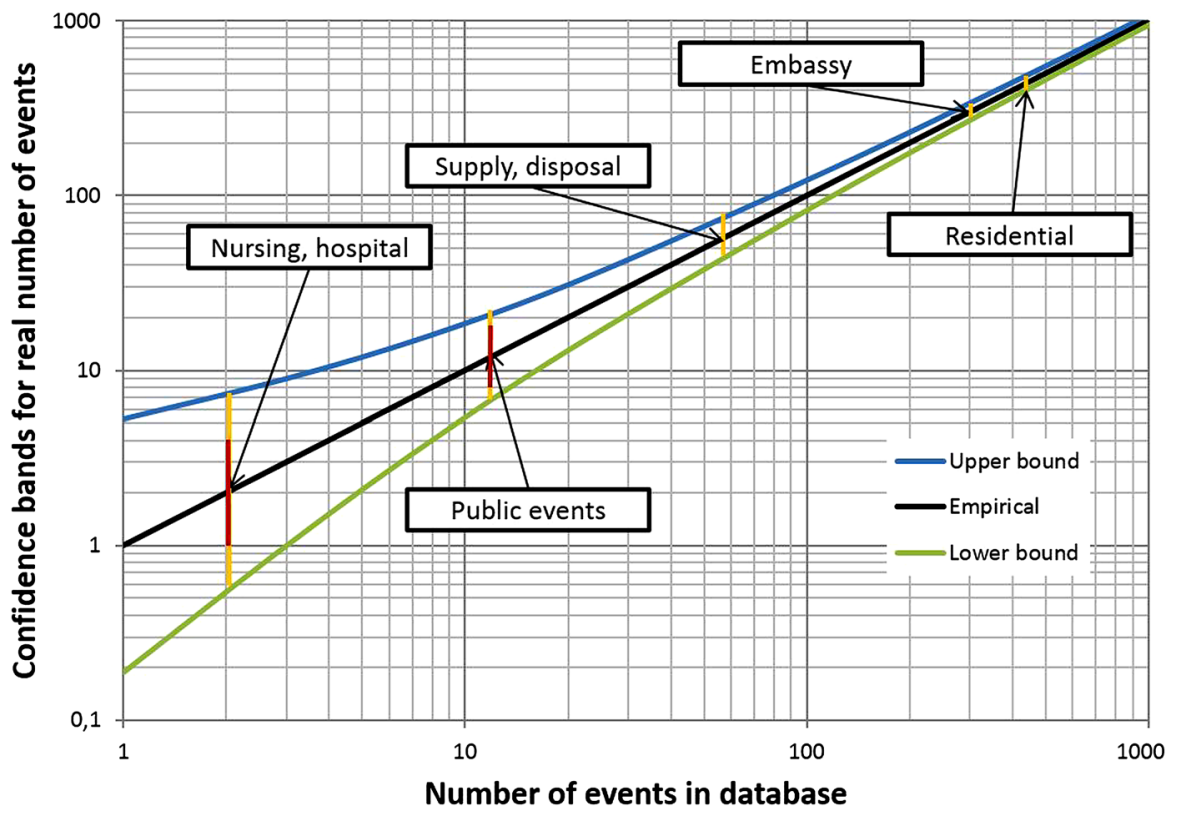

Fig. 4 Upper and lower confidence bands in terms of real numbers of events $x$ (yellow) with a proportion $1-\alpha$ of $95 \%$ according to (14). As an example, the figure shows multiplicative accuracy bands with accuracy $a_{\text {Susceptibility }}$ chosen to be equal to 2 and 1.5 (red) for "Nursing, Hospital" and "Public events" according to (15), respectively 
After showing how to determine the quantity of event data that suffices to achieve a defined accuracy of the empirical susceptibility when using the binomial or Poisson distribution, Sect. 5.3 achieves the same objective for the vulnerability using a modified Pareto distribution.

\subsection{Accuracy of Consequence, Vulnerability}

Determining the accuracy of the consequence and vulnerability quantities of Eqs. (6)-(8) is more complex, because a more complex distribution must be applied to assess the consequences in case of an attack. In contrast to the event frequency where the binomial distribution is chosen (event happens or not), the consequences have a wider range of values.

It is well known for the number of fatalities or injuries of severe events, that the power-law distribution, especially the Pareto distribution is a good estimation for reality, see for example Clauset et al. (2007). The power law is a heavy-tailed distribution: the tail is not exponentially bounded. It is also characteristic for power laws with exponent $k$, that they form straight lines with the respective slope $k$ in double-logarithmic diagrams.

Compared to Clauset et al. (2007), which focused on the frequency of severe terrorist events, this paper is not only interested in the tail of the distribution. In the present case, the average numbers of consequences over a wide range of consequences and estimates of their accuracy are required. However, it is well known that the power-law distribution is only a good estimation for more severe terrorist events (Clauset et al. 2007). Using the maximum likelihood method, only distributions with a variance of $\infty$ are obtained. Consequently, no assessment of the quality of the results is possible. Hence, another method is proposed to approximate the empirical data with a modified Pareto distribution. The Pareto distribution is truncated and combined with an empirical distribution for small consequence numbers.

The modified Pareto distribution is split up into three parts. For the first part with consequence numbers $x<x_{\min }$, the empirical numbers received from TED are used. This is done for a range from zero to the order of 10-50 of the consequence measure (e.g., number of injuries), where sufficient empirical data are available. In this case, the empirical density probability is obtained by dividing for each number of events (e.g., the number of events with 0 casualties in case of an event) for which the empirical density distribution is used, by the total number of events with the given combination of attack and building type(s) of interest.

For the second part $x_{\min } \leq x \leq x_{\max }$, the Pareto distribution is used for the consequence numbers. Normally, the Pareto distribution does not have a maximum value $x_{\max }$; however, the number of fatalities and injuries is certainly limited by the number of people on earth, and therefore, an upper limit is introduced. This is done for different $x_{\min }$ and with two different methods to determine the parameters of the modified Pareto distribution. The third part is a vanishing distribution.

In summary, with the slope $k>0$, we have the modified Pareto density distribution function: 


$$
f(x)= \begin{cases}P_{\text {empirical }}(X=[x]) & \text { if } x<x_{\min } \\ c_{\text {norm }} \frac{k}{x_{\min }}\left(\frac{x_{\min }}{x}\right)^{k+1} & \text { if } x_{\min } \leq x \leq x_{\max } \\ 0 & \text { if } x>x_{\max }\end{cases}
$$

where

$$
c_{\text {norm }}=c P_{\text {empirical }}\left(X \geq x_{\min }\right) \text { with } c=\frac{1}{\int_{x_{\min }}^{x_{\max }+1} \frac{k}{x_{\min }}\left(\frac{x_{\min }}{x}\right)^{k+1} \mathrm{~d} x}=\frac{1}{1-\left(\frac{x_{\min }}{x_{\max }+1}\right)^{k}} .
$$

In the case of the assessment of the statistical accuracy for the vulnerabilities (consequences), $x$ is the vulnerability quantity. With this definition, the following coherence holds:

$$
P(X=n)=P(n \leq x<n+1) .
$$

In Sects. 5.3.1 and 5.3.2, the slope $k$ of the distribution is determined for different partitioning (binning) numbers $x_{\min }$ of the distribution using two different methods.

\subsubsection{Maximum Likelihood}

Using the maximum likelihood method, as done in Clauset et al. (2007), Table 3 shows for different $x_{\min }$ [the lower bound where the analytical Pareto distribution function is used in Eq. (16)] the values for the slope $k$. For this method, the value of $x_{\max }$ does not matter as long as it is higher than the highest empirical value.

\subsubsection{Expectation and Variance}

Next, the expectation and variance method is applied to determine the best value for the slope $k$ for the Pareto-part of the consequence distribution. With the density of Eq. (17), the expectation $E$ of the second part can be determined as:

$$
\begin{aligned}
E(X) & =c \int_{x_{\min }}^{x_{\max }+1} k\left(\frac{x_{\min }}{x}\right)^{k} \mathrm{~d} x=c\left[-\frac{k}{k-1} \frac{x_{\min }^{k}}{x^{k-1}}\right]_{x_{\min }}^{x_{\max }+1}=c\left(\frac{k x_{\min }}{k-1}-\frac{k}{k-1} \frac{x_{\min }^{k}}{\left(x_{\max }+1\right)^{k-1}}\right) \\
& =c \frac{k x_{\min }}{k-1}\left(1-\left(\frac{x_{\min }}{x_{\max }+1}\right)^{k-1}\right) .
\end{aligned}
$$

Table 3 Slope $k$ for different consequences and different lower bounds $x_{\min }$ of the truncated Pareto distribution, determined with the maximum likelihood method

\begin{tabular}{llllllllll}
\hline$x_{\min }$ & 1 & 2 & 3 & 4 & 5 & 10 & 15 & 20 & 30 \\
\hline$k$ for fatalities & 0.914 & 1.032 & 1.084 & 1.123 & 1.148 & 1.324 & 1.344 & 1.343 & 1.381 \\
$k$ for injuries & 0.558 & 0.712 & 0.799 & 0.851 & 0.869 & 1.059 & 1.202 & 1.314 & 1.418 \\
$k$ for casualties & 0.535 & 0.675 & 0.756 & 0.814 & 0.842 & 0.985 & 1.096 & 1.175 & 1.272 \\
\hline
\end{tabular}

Casualties comprise injuries and fatalities 
To model the reality in a good way, Eq. (20) is set equal to the empirical expectation.

The method also includes to require that the distribution has the same variance as the empirical variance. To obtain the variance of the modified Pareto distribution, three cases are distinguished:

$$
\begin{aligned}
E\left(X^{2}\right) & =c \int_{x_{\min }}^{x_{\max }+1} k \frac{x_{\min }^{k}}{x^{k-1}} \mathrm{~d} x \\
& = \begin{cases}c\left[-\frac{k}{k-2} \frac{x_{\min }^{k}}{x^{k-2}}\right]_{x_{\min }}^{x_{\max }+1}=c \frac{k x_{\min }^{2}}{k-2}\left(1-\left(\frac{x_{\min }}{x_{\max }+1}\right)^{k-2}\right) & \text { if } k>2 \\
c\left[2 x_{\min }^{2} \ln x\right]_{x_{\min }}^{x_{\max }+1}=2 c x_{\min }^{2}\left(\ln \left(x_{\max }+1\right)-\ln \left(x_{\min }\right)\right) & \text { if } k=2 \\
c\left[-\frac{k}{k-2} \frac{x_{\min }^{k}}{x^{k-2}}\right]_{x_{\min }}^{x_{\max }+1}=c \frac{k x_{\min }^{2}\left(\left(\frac{x_{\max }+1}{x_{\min }}\right)^{2-k}-1\right)}{2-k} \text { if } k<2 .\end{cases}
\end{aligned}
$$

Then, the variance is obtained with the well-known identity:

$$
\operatorname{Var}(X)=E\left(X^{2}\right)-(E(X))^{2} .
$$

For the case $k=2$, the variance according to Eq. (22) reads $\operatorname{Var}(X)=$ $2 \ln \left(x_{\max }+1\right)-4\left(1+\frac{1}{x_{\max }+1}\right)^{2}$ for $x_{\min }=1$ and $k=2$. A slope of $k \approx 2$ has often been used in frequency-severity distributions of wars (Richardson 1948; Cedermann 2003), in particular, for their tails.

As in Clauset et al. (2007), the scaling law can also be applied to empirical terrorism data. It is important to determine the slope empirically (Clauset et al. 2007). This holds especially true, when looking at the variances, which are of key interest in the present approach: $k=2$ proposed in Taleb (2008) seems to be an unrealistic value: Setting $x_{\min }=1$ and $x_{\max }=7 \times 10^{9}$, the number of people on earth, $\operatorname{Var}(X)=41$ is obtained for fatalities, but the empirical value derived from TED is $\operatorname{Var}(X)=418$. Therefore, for given $x_{\min }$, the values of $k$ and $x_{\max }$ are determined by solving the Eqs. (20)-(22) numerically, after inserting the empirical values for expectation and variance derived from TED, see Table 4.

\subsubsection{Comparison and Conclusion}

To compare the results obtained with the two different methods, the KolmogorovSmirnov goodness-of-fit test is used. This test is done for all possible $x_{\min }$ for the maximum likelihood and the method of expectation and variance of Sects. 5.3.1 and 5.3.2, respectively. The method of Sect. 5.3.2 delivers better $p$ values for the Kolmogorov-Smirnov test. A comparison of the results as obtained for the root vertex "Urban area" can be found in Table 5. 
With the method of expectation and variance $(\mathrm{EV})$ for fatalities and $x_{\min }=11$, the best $p$ value is obtained. Hence, the chosen distribution has the density of Eq. (17) with the parameters $x_{\min }=11, k=1.18$ and $x_{\max }=421$ from Table 5. Altogether, the EV method delivers better results. In all cases, there is no reason to reject the truncated Pareto model ( $p$ value $\geq 0.05$ ), independent of the method of parameter determination.

The analysis in Sect. 5.3 determines the multiplicative accuracies for the vertexes of Fig. 3 with respect to consequences. Using the values for $k, x_{\min }$ and $x_{\max }$ of the

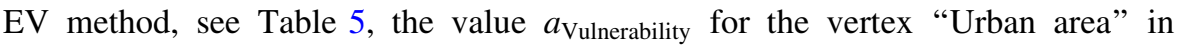
Table 6 is obtained via bootstrapping. The other vertexes are obtained correspondingly.

To receive the accuracy for a specific consequence $T_{n}^{\text {consequence }}$ of a specific threat $T_{i}^{\text {threat }}$ for a specific building type $T_{l}^{\text {building }}$ in a specific region $G_{h}$, the EV method, Kolmogorov-Smirnov test and bootstrapping must be carried out for the desired combination.

Table 6 shows the multiplicative accuracy for the root "Urban area" and the example vertexes "Embassy" and "Public Events" in Western Europe. These values are also further discussed in Sect. 6.

In summary, as shown exemplarily in Table 6, when using the definitions of Sects. 3 and 4, the empirical prediction of the vulnerability is worse than the prediction of the susceptibility. To receive for $\alpha \leq 5 \%$ a multiplicative accuracy of 2 , the database must hold about 200 events (in contrast to 8 for the susceptibility) for the regarded vertex. For Western Europe, these data are not available for many vertexes of Fig. 3. See, for instance, the middle and the last columns of Table 6 for examples where the data are available and unavailable, respectively.

Table 4 Slope $k$ and $x_{\max }$ for different $x_{\min }$ and different consequences for a modified Pareto distribution, see Eq. (17), determined with the method of expectation and variance

\begin{tabular}{llllllllll}
\hline$x_{\min }$ & 1 & 2 & 3 & 4 & 5 & 10 & 15 & 20 & 30 \\
\hline$E(X)$ for fatalities & 7.26 & 10.51 & 13.66 & 16.62 & 19.51 & 31.31 & 43.7 & 55.8 & 76.82 \\
$E(X)$ for injuries & 17.17 & 19.91 & 23.14 & 26.54 & 30.35 & 43.16 & 54.71 & 65.93 & 90.06 \\
$E(X)$ for casualties & 19.55 & 22.6 & 26.02 & 29.38 & 33.16 & 47.85 & 60.49 & 72.75 & 97.15 \\
$\operatorname{Var}(X)$ for fatalities & 418 & 604 & 791 & 971 & 1148 & 1889 & 2661 & 3375 & 4437 \\
$\operatorname{Var}(X)$ for injuries & 6501 & 7554 & 8845 & 10,258 & 11,892 & 17,818 & 23,853 & 30,301 & 45,830 \\
$\operatorname{Var}(X)$ for casualties & 7159 & 8270 & 9560 & 10,869 & 12,378 & 18,595 & 24,481 & 30,647 & 44,149 \\
$k$ for fatalities & 0.875 & 0.987 & 1.039 & 1.074 & 1.097 & 1.227 & 1.217 & 1.209 & 1.208 \\
$k$ for injuries & 0.73 & 0.863 & 0.94 & 0.99 & 1.019 & 1.183 & 1.29 & 1.366 & 1.442 \\
$k$ for casualties & 0.682 & 0.806 & 0.879 & 0.931 & 0.963 & 1.101 & 1.197 & 1.264 & 1.349 \\
$x_{\max }$ for fatalities & 297 & 344 & 366 & 382 & 391 & 453 & 438 & 435 & 432 \\
$x_{\max }$ for injuries & 1606 & 2031 & 2362 & 2623 & 2790 & 4123 & 5565 & 7045 & 9028 \\
$x_{\max }$ for casualties & 1439 & 1750 & 1986 & 2186 & 2320 & 3057 & 3784 & 4439 & 5495 \\
\hline
\end{tabular}

$E(X)$ and $\operatorname{Var}(X)$ are the empirical values derived from TED for the second part of the distribution (17) for different $x_{\min }$ 
Table 5 Comparison of the maximum likelihood method (ML) with the method of expectation and variance $(\mathrm{EV})$

\begin{tabular}{llllllrll}
\hline & $N$ & $E(X)$ & $\operatorname{Var}(X)$ & $N_{\text {tail }}$ & $x_{\min }$ & $x_{\max }$ & $k$ & $p$ value \\
\hline ML fatalities & 16,247 & 2.12 & 132 & 682 & 11 & 500 & 1.28 & 0.10 \\
ML injuries & 15,933 & 6.97 & 1846 & 579 & 40 & 5000 & 1.48 & 0.09 \\
ML casualties & 16,201 & 9.29 & 3209 & 669 & 46 & 5254 & 1.41 & 0.65 \\
EV fatalities & 16,247 & 2.2 & 138 & 682 & 11 & 421 & 1.18 & 0.7 \\
EV injuries & 15,933 & 7.09 & 2758 & 579 & 40 & 10,277 & 1.48 & 0.1 \\
EV casualties & 16,201 & 9.17 & 3453 & 685 & 45 & 7015 & 1.44 & 0.33 \\
\hline
\end{tabular}

$N$ and $N_{\text {tail }}$ depict the number of events in the overall and the second non-vanishing part of the distribution for the root vertex "Urban area"

Table 6 Multiplicative accuracy $a_{\text {vulnerability }}$ of the vulnerability and $a_{\text {Risk }}$ of the risk for an error level $\alpha \leq 5 \%$ for fatalities (Fat), injuries (Inj) and Casualties (Cas) for different vertexes of Fig. 3 and different geographic regions compared with the multiplicative accuracies for the corresponding accuracies of the susceptibilities $a_{\text {Susceptibility }}$

\begin{tabular}{|c|c|c|c|c|c|c|c|c|c|}
\hline $\begin{array}{l}\text { Geographic region } \\
\text { Building type } \\
\text { Accuracy } a_{\text {Susceptibility }}\end{array}$ & \multicolumn{3}{|c|}{$\begin{array}{l}\text { Worldwide } \\
\text { Urban area } \\
1.02\end{array}$} & \multicolumn{3}{|c|}{$\begin{array}{l}\text { Western Europe } \\
\text { Embassy } \\
1.12\end{array}$} & \multicolumn{3}{|c|}{$\begin{array}{l}\text { Western Europe } \\
\text { Public events } \\
1.75\end{array}$} \\
\hline Consequence & Fat & Inj & Cas & Fat & Inj & Cas & Fat & Inj & Cas \\
\hline 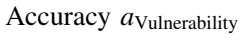 & 1.09 & 1.16 & 1.12 & 1.79 & 1.74 & 1.85 & 13.2 & 10 & 8.6 \\
\hline Accuracy $a_{\text {Risk }}$ & 1.09 & 1.16 & 1.12 & 1.81 & 1.76 & 1.87 & 14 & 10.7 & 9.2 \\
\hline
\end{tabular}

The challenge of empirical-statistical consequence estimates could be resolved by a more precise definition of the events with respect to the type and location of attack as well as physical structure information for the building. E.g., one could introduce net explosive quantity ranges, categories like "event location close to building" and building types like "Masonry building". However, such data are not publicly available. That is why the empirical prediction of the consequences for risk assessment can only give a very broad indication. It also prevents to distribute the information extracted from the data to be more balanced between the susceptibility and vulnerability quantities.

In consequence, for detailed analysis, further assessments including physical models for consequences, like mechanical models of structural resistance or biomechanical damage should be applied. Furthermore, the possible attack locations must be defined as well as the distribution of possible events on these locations, e.g., increasing event densities close to buildings.

\subsection{Accuracy of Averaged Risk Quantities}

To determine the accuracy of the risk quantities of Eqs. (9)-(12), the classical treatment for the propagation of uncertainty is used. The accuracies of Sects. 5.2 
and 5.3 have a symmetric error bar in a log-scaled metric. Using error propagation, it is possible to derive that the multiplicative accuracy for the risk quantities reads:

$$
a_{\text {Risk }}=\sqrt{\left(\ln a_{\text {Susceptibility }}\right)^{2}+\left(\ln a_{\text {Vulnerability }}\right)^{2}} .
$$

For the same examples as in the last section, this results in the values presented in Table 6 for the multiplicative accuracy $a_{\text {Risk }}$.

Table 6 shows that the accuracy $a_{\text {Risk }}$ is nearly the same as the accuracy $a_{\text {Vulnerability }}$. This is not surprising, because in all cases, $a_{\text {Vulnerability }}$ is much greater than $a_{\text {Susceptibility }}$, so that $a_{\text {Risk }}$ is almost only influenced by $a_{\text {Vulnerability }}$. More detailed locally resolved susceptibilities along with physics-based consequence models are, thus, desirable also to improve the accuracy of risk calculations.

\section{Example of Empirical Urban Area Terrorism Susceptibility, Vulnerability and Risk Assessment and Improvement}

Figure 5 shows the results of the application of the methodology to an urban sample area using the VITRUV-EDEN software, which implements the approach as described in Sects. 4 and 5.1. The analysis is based on data of Western Europe from 1969 to 2008. Of course, in practice, other time intervals could also be used, in particular, shorter time intervals that reach up to the point of time of assessment. Also, much more advanced estimators could be used going beyond homogeneous smoothing.

However, in particular, the following arguments can be listed for the chosen approach. Even for the rather simple statistical approach, the presented statistical accuracy assessments for averaged susceptibility, vulnerability and risk quantities hold true only for sufficient event data, as discussed in Sect. 5. The chosen approach can be communicated to urban planners due to its reasonable level of complexity fostering informed decision-making versus black-box assessment when using complex methods. To get an impression of the ranges (minima and maxima) and orders of magnitudes, other worldwide regions can also be used for assessment as well as other time intervals. For instance, worst and best combinations of time intervals and urban regions can be used. For an assessment of urban scenarios, often relative comparisons suffice. Buildings have a very long life-cycle time-span. Hence, susceptibility, vulnerability and risk can be expected to change significantly several times during this time span. Planning has to consider all such phases.

Figure 5 shows how critical buildings can be detected using various quantities and how the tool gives support for decision-makers to apply possible resilience enhancement measures in a very early stage of the urban planning process. Figure 5 includes all building types of Fig. 3 which lead, respectively, to the highest and the lowest value of empirical susceptibility, vulnerability and averaged risk.

Concerning the empirical susceptibility in terms of events per building per year according to Eq. (4) (Fig. 5a, upper left), the building with the usage "Embassy" 

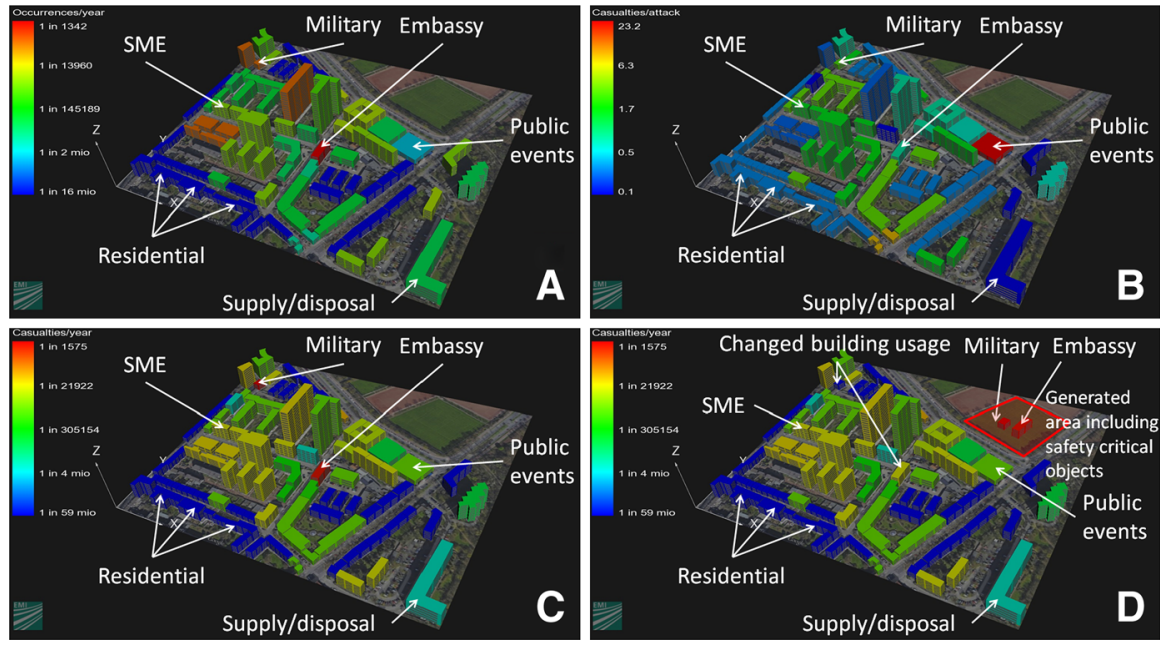

Fig. 5 Visualization of susceptibility (a), vulnerability (b) and empirical risk (c) for a sample urban area to identify weak spots with respect to terrorism. (d) A modified urban design where the critical objects are relocated to generate a larger distance to neighbour urban objects

has the highest value in contrast to "Residential" buildings, which have the lowest value.

Figure $5 \mathrm{~b}$ shows that for the empirical vulnerability in terms of casualties per event according to Eq. (7), the vertex or building type "Public event", which could be a concert hall, for example, has the highest empirical vulnerability value. The lowest casualties per event are predicted for the vertex "Supply, disposal", e.g., waterworks. It is consistent that the latter building type has a low value for casualties per event, because a threat against such a building typically aims to damage the supply and not to harm many people.

One observes that the susceptibility and vulnerability assessments identify different buildings with highest and lowest values, respectively. In urban planning scenarios, the susceptibility information can be used to assess likely locations of events, giving guidance to the question of where to expect terrorist events and where to prevent them, e.g., using urban designs that control access. The vulnerability information supports the answer to the question, what are the consequences in case of an event (what-if assessment), independently of the question, how susceptible the building or infrastructure is with respect to terrorist events. Taking account of this assessment options, for instance, it could be decided that independent of the risk assessment, very high susceptibilities as well as very high vulnerabilities must be avoided.

Visualization $\mathrm{C}$ in Fig. 5 shows that for the risk in terms of casualties per year per building, again, the vertex "Residential" has the lowest value. The highest value is obtained by "Military" which has medium consequences and a high frequency. The "Embassy" with a higher frequency has a lower vulnerability resulting in a bit smaller risk. This reflects that embassy facilities have higher protection standards. This is an example where the risk quantity identifies again a new most critical 
building type when compared to the pure susceptibility and vulnerability assessment! For instance, it could be decided that risks of buildings or infrastructure beyond a certain level must be mitigated by urban design on plan level.

To get an impression how risk control could be achieved in an informed way, this example is further elucidated. An often-used international standard for individual acceptable risk of mortality per year is one in a million (Proske 2008), the de minimis risk $R_{\mathrm{de} \text { minimis }}=1.0 \times 10^{-6} a^{-1}$. The average casualties values per year in the visualization $\mathrm{C}$ in Fig. 5 are not individual annual risk values but annual collective casualty numbers per building. Even when assuming that the collective risk $R_{\text {ind,embassy }}$ is equally shared between all members $N_{\text {embassy }}$ of the embassy, i.e., $R_{\text {col, embassy }}=N_{\text {embassy }} R_{\text {ind,embassy }} \leq N_{\text {embassy }} R_{\text {deminimis }}$, only if the risk can be shared between more than $N_{\text {embassy }} \geq R_{\text {col, embassy }} / R_{\text {deminimis }} \approx 500$ persons, it is below the de minis risk. Hence, the computed annual collective risk would not be acceptable when applying the well-known de minimis risk criteria to casualties. In Proske (2008), in particular, many other individual annual risk quantities and values can be found which an urban planner can use for comparison. It is up to the planners to evaluate the analysis results.

The empirical risk approach allows users to detect likely hotspots using susceptibilities, vulnerabilities and/or risks. In a further step, it can be discussed on plan level how to resolve these issues. For instance, critical objects could be relocated to avoid the overlapping with uncritical objects in the neighbourhood and to locally better control risks.

Visualization D in Fig. 5 shows an option to rearrange the urban scenario by generating a subarea for the highly endangered buildings of visualization $\mathrm{C}$. Grouping high-risk buildings together makes it easier to protect these buildings. For instance, whole quarters like embassy quarters could be blocked for cars. By shifting high-risk buildings, neighbouring effects for public buildings, for instance, schools or kindergartens, can be minimized. In the present example, it is assumed that the quantities close to the security area do not change. Hence, the remaining quarter has no new critical buildings. It is assumed that the terrorist events do not occur at substitute locations. However, the approach can be used to identify substitute critical buildings close to the security area. They can be expected to be buildings with high susceptibility, vulnerability or/and risk close to the secure area or the highest buildings outside the secure area (Coaffee 2009).

Another option to improve the security of the urban area, at least with respect to personnel in buildings, could be achieved using, e.g., physical access control and physical enhancement measures for the endangered buildings. In the present approach, the effect of these enhancement measures could enter as scaling factors, as described in Sect. 4.2 to modify susceptibilities and vulnerabilities. For the "Small and Medium sized Enterprise" (SME) buildings, these scaling factors could, for instance, also stand for efficient surveillance systems, the presence of active security guards or a high rate of police patrols. For the "Embassy", they could represent enhanced structural response against blast using more resistant innovative materials or the use of bollards to control the physical access to the "Embassy" by vehicles. Another perspective on selecting improvement options is to choose 


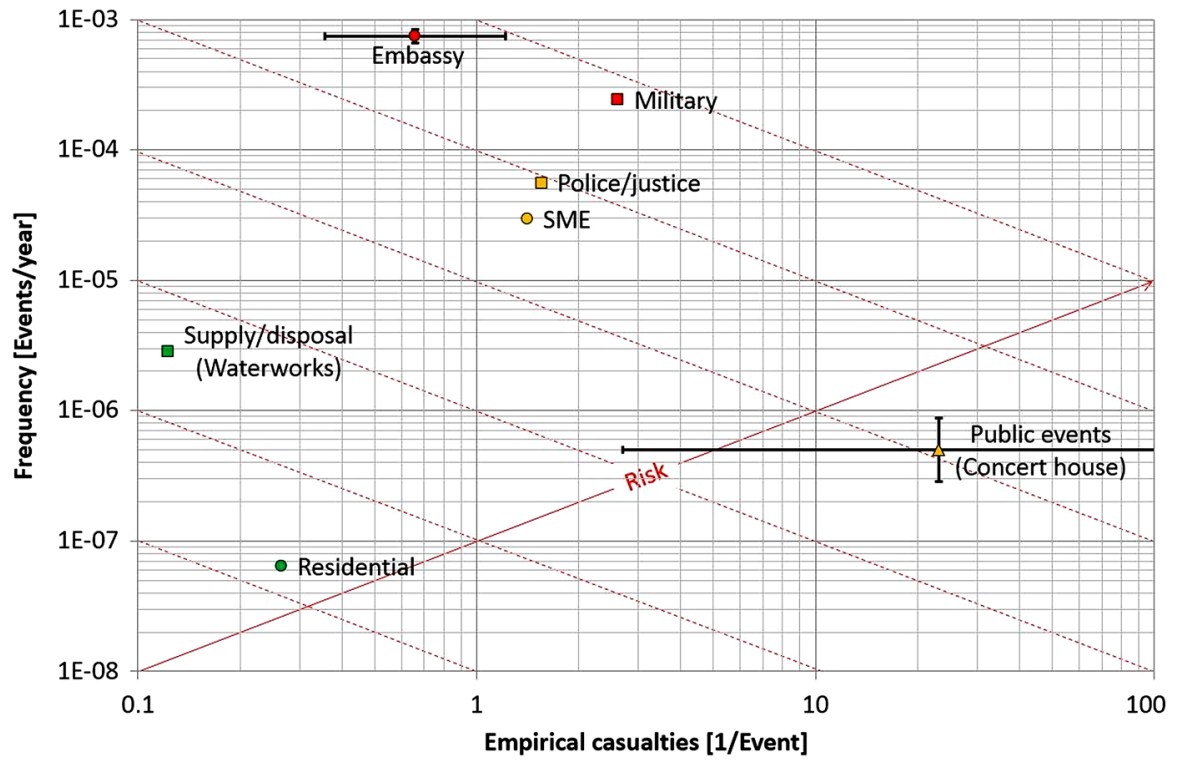

Fig. 6 Empirical frequencies (susceptibility) and casualties (consequence) of a selection of some building types of the example urban area of Fig. 5 as resulting from the terror event database analysis. In addition, the product of both, the empirical risk, can be read off by means of the diagonal red dashed lines. For the examples "Embassy" and "Public events", the multiplicative error bars of the empirical frequency and consequences are shown

detailed designs that make, for instance, selecting a prominent pair, embassies as low susceptible as public event locations and public event locations as low vulnerable as embassy buildings.

The scaling factors have to be used carefully, because determining them for a specific enhancement measure needs a lot of expert knowledge. If empirical consequence models are replaced by physical-engineering models, the modelling of enhancement measures (i.e., increased resistance of walls/windows or reduced blast overpressure by bollards) will be much more quantitatively accessible. This also holds true for physical access control means, e.g., bollards preventing the access of larger vehicles, and hence, e.g., very high explosive net quantities.

Figure 6 shows the empirical susceptibility (frequency), vulnerability (consequences) and risk of a selection of some building types of the urban area of Fig. 5. The sample accuracy results for the two non-root vertices for Table 6 discussed in Sect. 5 are also depicted.

The accuracy analysis of Sect. 5 shows that the results of the example calculation A in Fig. 5 are most accurate. For all defined building types, the multiplicative accuracy for the prediction of the susceptibility in terms of number of events per year is smaller than 2. The worst accuracy of the example is obtained by "Public events" (1.75). The best one is obtained by "Residential" (1.1) followed by "Embassy" (1.12).

The accuracy for the prediction of vulnerability in terms of casualties per event (attack) per building and risk in terms of casualties per building per year is 
considerably less reliable. For "Embassy" (1.85, respectively, 1.87), see Table 6, it is still not bad. The accuracy for the category "Public events" (8.6, respectively, 9.2 ) is quite bad. This means that it is only known that the mean value or expectation of 23.2 for the casualties per attack for "Public events" lies within the confidence interval of 2.7-200 with a probability of $95 \%$ ! This is still an indication that the number of casualties for an event at "Public events" is, on average, very high, but the interpretation has to be done very carefully. For instance, the urban design as well as the precise scenario setting can be expected to strongly influence the vulnerability. See, also, Fig. 1 for the multiplicative error bars.

During the development of the application software tool, several urban planners as end-users were included. Concerning the feedback to the empirical analysis, it was stated that the tool, which visualizes the quantitative empirical-statistical analysis, is very useful to obtain a quick assessment of an urban area. Weak spots are marked in a sufficiently intuitive way and the users can consider them in their planning process to evaluate and enhance the security of the urban area. In the planlevel phase, possible changes are much cheaper than in later planning phases. Also, the empirical-statistical analysis allows to concentrate the finer resolved and more resource-intensive quantitative engineering-simulative susceptibility, vulnerability and risk assessment to areas only that are identified as critical, as indicated in Sects. 2 and 3.

\section{Conclusions and Outlook}

The paper presented a general quantitative approach for the evaluation of terroristic threats in urban areas on plan level. Based on empirical-historical data, susceptibility, vulnerability and, finally, risk quantities are defined for buildings and infrastructure. It was derived how the approach relates to generalized frequencies (probabilities) and consequences (damage) quantities as well as further extensions using engineering and simulative approaches on detail level. The paper discussed how the present overall quantitative statistical-empirical approach relates to broader interdisciplinary concepts of susceptibility, vulnerability and risk.

A software-based solution visualizes the results within a three-dimensional user interface. The assessment on plan level gives support to identify, to discuss and, potentially, to redefine (e.g., by changing the usage), to redesign (e.g., using physical reinforcement), to change the context (e.g., by access control) or to relocate buildings and infrastructure identified as critical. The assessment quantities indicate where security and safety measures are likely to be relevant. In particular, the empirical-historical data of three different worldwide regions can be used to assess benign, average and worst-case overall developments with respect to the terroristic threat level.

Analysing terrorist risks in isolation would not help to design better urban plans. The proposed approach should be used by simultaneously taking into account other criteria which are equally important for urban planning, such as cost, infrastructure availability, ease of transportation and logistics, environmental footprint, urban 
quality of living, etc. The approach could enter into an overall optimization approach taking into account the mentioned points.

The approach supports to plan more resilient cities which are less susceptible and less vulnerable (more robust) and, hence, more sustainable with respect to terroristic threats. The application allows the consideration of security and safety enhancement measures at early stages of the urban planning processes, where changes are not yet too expensive.

A further advantage of the fast empirical assessment is that whole urban areas and not only single critical infrastructures or buildings can be evaluated. The quantitative risk assessment for urban areas forms the basis to decide where and, to some extent, also which resilience enhancement measures are essential.

The paper focused strongly on the empirical-historical statistical approach. A hierarchical categorization of the empirical data based on the usage of buildings and infrastructure was defined to apply the empirical data analysis in a reliable way on urban environments. This semantic categorization allows the controlled use of upper vertexes if a layer has limited empirical data.

Assuming reasonable distribution types for the frequency and the consequences, respectively, the accuracy of the quantities derived from the empirical data was analysed. The analysis was conducted on the assumption that the historical data are complete and the number of buildings and infrastructure at risk known. These are realistic assumptions for representative historical event data sets and known numbers of building types.

The statistical results showed rather accurate statistical data for the frequency of events. The present data for the vulnerabilities (consequences), e.g., casualties per event are decisively less accurate but can still be used as a first indicator. In both cases, it was shown how to determine and control the accuracy of the empirical susceptibility, vulnerability and risk quantities using symmetric multiplicatively defined confidence intervals as appropriate for the risk and resiliency assessment context.

In particular, it was shown that a modified Pareto density distribution can be well used to represent the empirical consequence distributions. The modified Pareto distribution is composed of an empirical distribution and a truncated classical Pareto distribution.

With the help of the distributions for combinations of worldwide region type, building type and threat type, as defined in a directed graph (arborescence), respectively, the expected susceptibility (frequency) and vulnerability (consequence) can be estimated along with multiplicative error intervals with a defined accuracy. It was found that typically already only 8 events suffice for the assessment of the susceptibilities, whereas ca. 200 are necessary for similar accurate results for the consequences. This holds true when requiring a multiplicative accuracy of 2 and an error level of $5 \%$, i.e., the true value lies with $95 \%$ in an interval given by the lower bound consisting of the expectation value divided by 2 and the upper bound by the expectation value multiplied by 2 .

For further analysis, in particular, of the vulnerability and risk quantities, it is recommended to use a refined quantitative risk assessment approach, for example, 
with physical consequence models, such as single-degree-of-freedom approaches, to overcome the rather weak empirical data basis.

The empirical risk analysis delivers rather reliable susceptibility values for single urban objects, which can provide the basis of the further quantitative assessment also of the susceptibilities. It is expected that the assumptions about the precise possible locations of threats (hazard sources) strongly influence the local susceptibilities (local frequencies of terrorist events) as well as the vulnerabilities and hence risk-related quantities, in particular, when defined in terms of local individual and collective risks. This would, in particular, allow to show neighbourhood effects, i.e., how high-risk buildings increase the vulnerabilities and risks in the neighbourhood. One first sample approach taking up the identified need of simulative-engineering and probabilistic quantification of physical consequences and damage effects is given in Fischer et al. (2016), showing that it allows for improved vulnerability and averaged risk assessment to enhance the resilience of urban areas.

Furthermore, it is expected to be challenging to find an adequate visualization of the uncertainties, which are expected to be overcome only in parts, also when using detailed information on the urban geometries and detailed building and infrastructure designs for further engineering-simulative-based refinement of vulnerabilities and averaged risks.

Finally, since the used empirical input data are rather limited, comprising only date, location, type of event, object at risk and two numeric consequence quantities, the present approach could also be easily extended to other natural, man-induced natural and man-made accidental hazards for which similar data are available, in particular, crime event data.

Acknowledgments The research leading to these results has partly received funding from the European Union Seventh Framework Programme FP7 under Grant Agreement No. 261741 and 313077. The authors gratefully acknowledge contributions and discussions with the partners of the VITRUV project, namely Crabbe Consulting Ltd, Provincia di Bologna, West Yorkshire Police, Schüßler Plan Ingenieurgesellschaft mbH, Dissing + Weitling architecture, TNO research laboratories, Downey Hynes Partnership, CEUSS of Sigmund Freud University Vienna, Decisio Economic Consulting, Thales Communications and Security and London Borough of Southwark as well as with partners of the EDEN project, namely Ainia, Astri Polska, Airbus Defence and Space, BAE Systems, Bruker UK, CBRNE Centre, CBRNE Ltd, ENEA, European Virtual Institute for Integrated Risk Management, Fondation pour la Recherche Strategique (FRS), Fraunhofer ICT, Fraunhofer INT, Hotzone Solutions BV, Indra Sistemas, S.A., INERIS, Interuniversity Chair in Law and the Human Genome, Istituto Affari Internazionali (IAI), LDIAMON, The Main School of Fire Service (SGSP), MDA, Microfluidic, Norwegian Defence Research Establishment (FFI), Nucletudes, OMNIDATA S.A., Przemysowy Instytut Automatyki i Pomiarłw PIAP, Robert Koch Institut (RKI), SAMU, SELEX ES, SICPA SA, Space Research Center, Tecnoalimenti, Universita Cattolica del Sacro Cuore, Universita catholique de Louvain (UCL), Center for Applied Molecular Technologies (CTMA), University of Reading, Public Health England.

\section{Appendix: Overview on Empirical Data Used}

This section provides an overview of the empirical data for terrorist events, which are implemented in the VITRUV software for risk assessment at plan level. Figures 7, 8 and 9 show that some categories have no values, i.e., no events or 


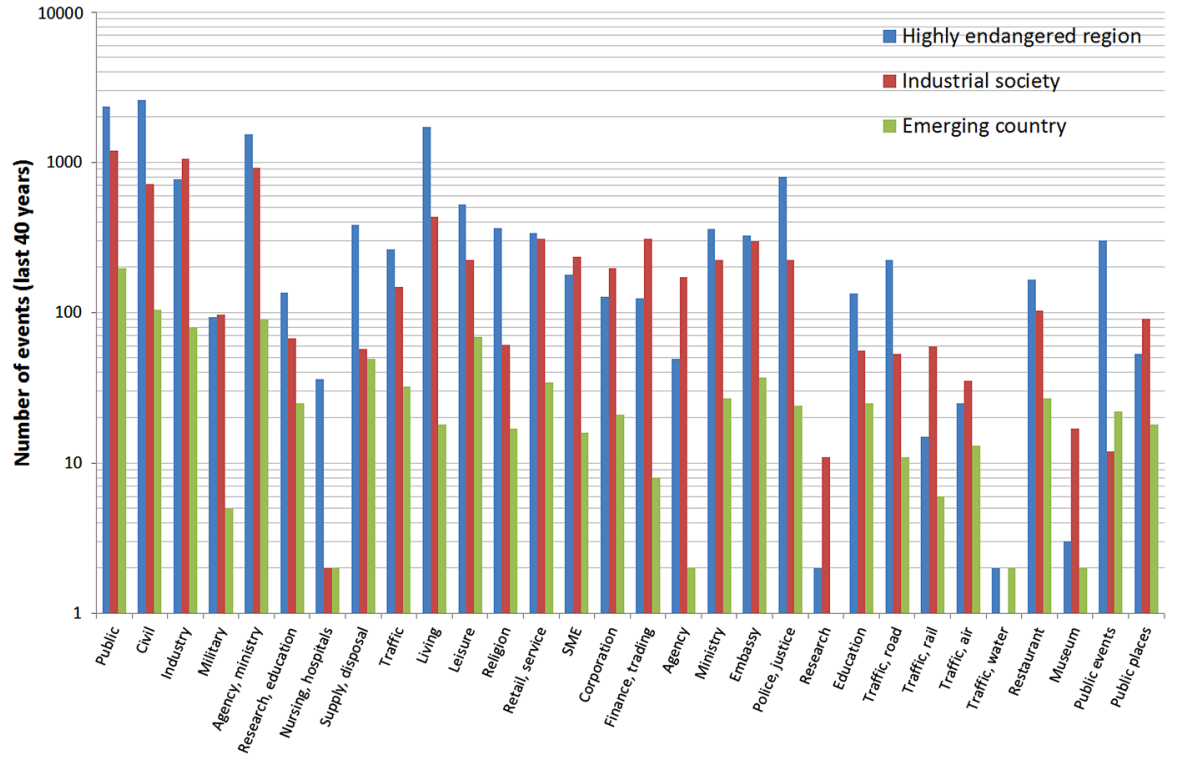

Fig. 7 Number of terrorist events for each considered category and region which are used in the VITRUV software

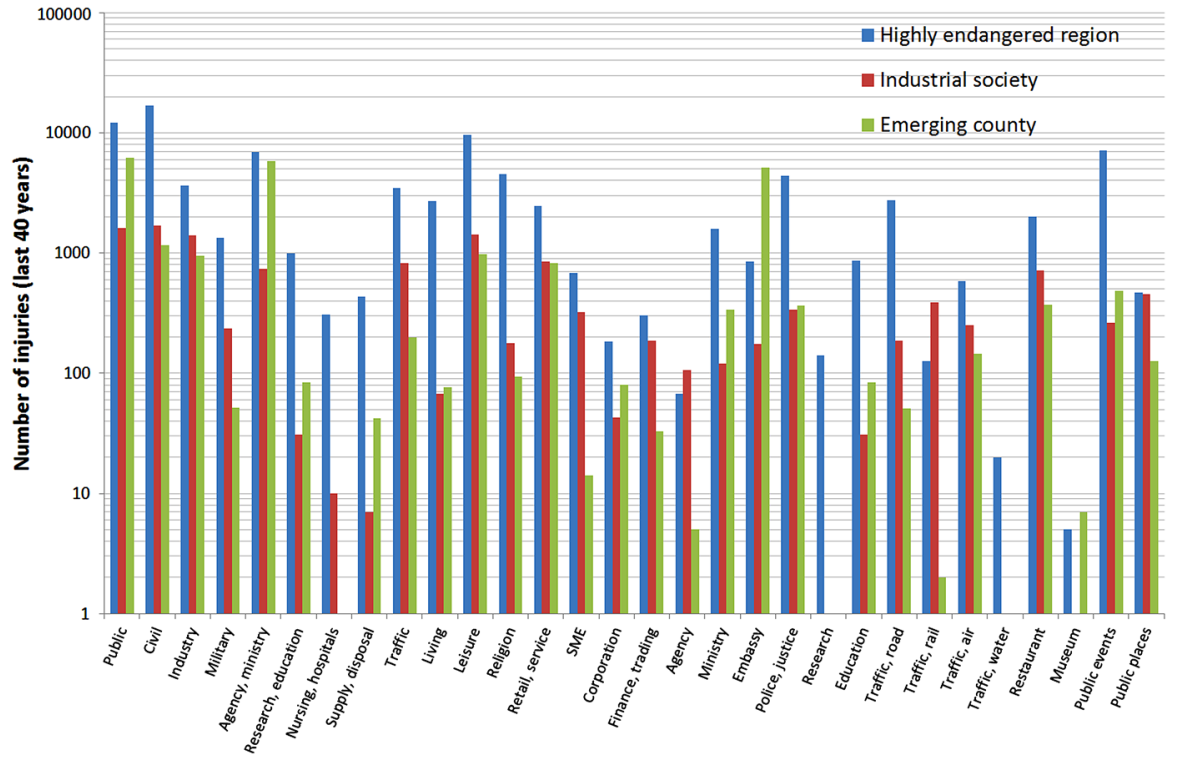

Fig. 8 Number of injuries for terrorist events for each considered category and region which are used in the VITRUV software 


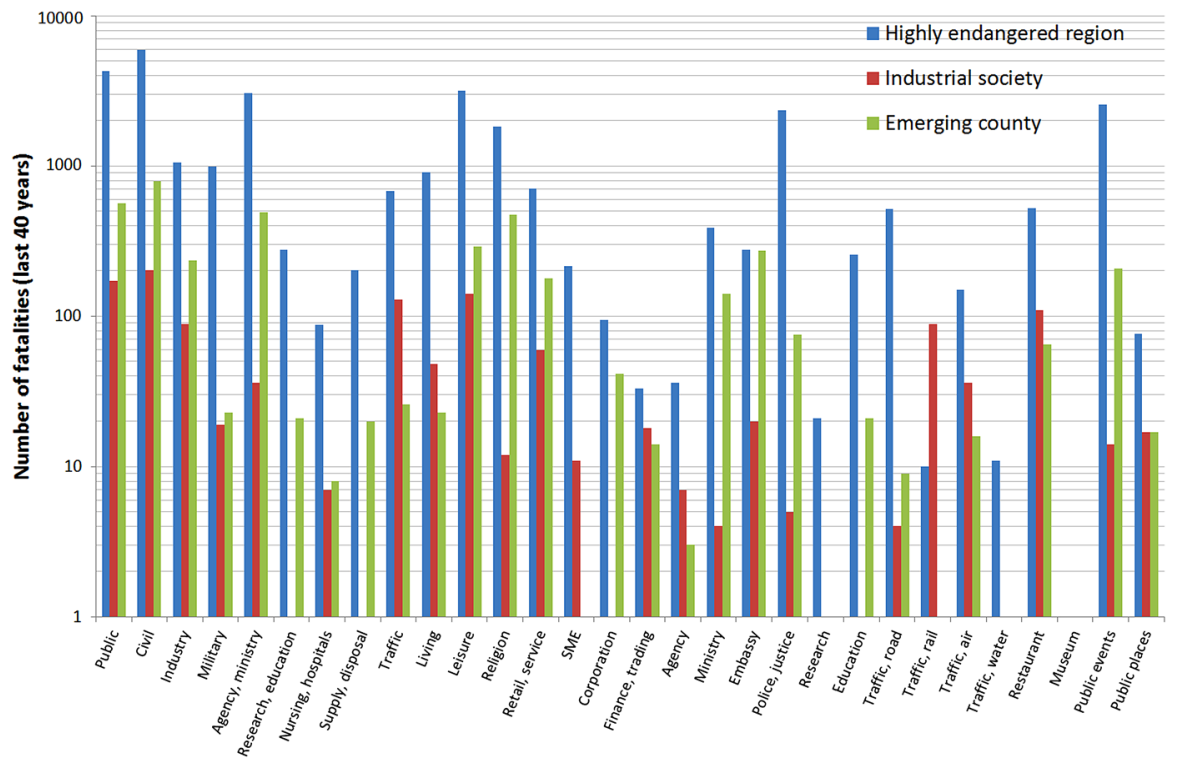

Fig. 9 Number of fatalities for terrorist events for each considered category and region which are used in the VITRUV software

consequences were available for these targets. In such a case or if the data set is too small, the VITRUV software automatically goes to the parent vertex of the arborescence, see Fig. 3, and uses the data of the superior urban category.

In the case of susceptibilities, also the number of total buildings for a special target category enters the empirical quantity. These numbers are based on estimates for Western Europe.

\section{References}

Adger WN (2006) Vulnerability. Glob Environ Change 16:268-281

Ale BJM (2002) Risk assessment practices in the Netherlands. Saf Sci 40(1-4):105-126

Aschmoneit T, Richter C, Gürke G, Brombacher B, Ziehm J, Häring I (2009) Parameterization of consequences of explosions. In: European safety and reliability conference (ESREL). CRC Press, Prague

Aschmoneit T, Häring I (2010) Resampling expert estimates for the consequence parameterization of explosions. In: Ale BJM, Papazoglou IA, Zio E (eds) European safety and reliability conference (ESREL). CRC press, Rhodes, p 49-56

BAE Systems (2015) EU project EDEN: end-user driven demo for CBRNE 2015 [cited 2016 14.07]. www.eden-security-fp7.eu/

BIM (2016) [cited 2016 14.07]. www.cadline.co.uk/Products/BIM

Ball RE (2003) The fundamentals of aircraft combat survivability analysis and design. AIAA education series. American Institute of Aeronautics and Astronautics (AIAA), Reston

Birkmann J (2007) Risk and vulnerability indicators at different scales: applicability, usefulness and policy implications. Environ Hazards 7(1):20-31

Branscomb LM (2006) Sustainable cities: safety and security. Technol Soc 28(1-2):225-234 
Cedermann L-E (2003) Modeling the size of wars: from billiard balls to sandpiles. Am Polit Sci Rev 97(1):135-150

CityGML (2016) [cited 2016 14.07]. www.citygml.org

Clauset A, Young M, Gleditsch KS (2007) On the frequency of severe terrorist events. J Conflict Resolut 51(1):58-87

Clopper C, Pearson ES (1934) The use of confidence or fiducial limits illustrated in the case of the binomial. Biometrika 26(4):404-413

Coaffee J (2008) Risk, resilience, and environmentally sustainable cities. Energy Policy 36(12):4633-4638

Coaffee J (2009) Terrorism, risk and the global city: towards urban resilience. Ashgate, Burlington

Cross JA (2001) Megacities and small towns: different perspective on hazard vulnerability. Glob Environ Change Part B Environ Hazards 3(2):63-80

Dorsch H, Bongartz A, Roßberg D (2013) Modelling of ballistic effects with regard to urban operations. In: Wickert M, Salk M (eds) 27th International symposium on ballistics. DEStech Publications, Inc., Freiburg, p 591-601

Dorsch H, Dörr A, Bongartz A (2014) Modelling of behind debris wall effects. In: Ames RG, Boeka RD (eds) 28th International symposium on ballistics. DEStech Publications, Inc., Atlanta, p 1462-1471

Edwards C (2009) Resilient nation. Demos, London

FEMA (2003) Reference Manual to Mitigate Potential Terrorist Attacks Against Buildings. In: U. S. Department of Homeland Security and Federal Emergency Management Agency (FEMA) (ed) Risk management series, U.S. Department of Homeland Security, Washington D.C. pp 1-420

FEMA (2011) Reference manual to mitigate potential terrorist attacks against buildings. In: U. S. Department of Homeland Security: Science and Technology Directorate; FEMA (Federal Emergency Management Agency) Risk Reduction Division (ed) Buildings and infrastructure protection series (BIPS), U.S. Department of Homeland Security, Washington D.C. pp 1-514

Fischer K, Siebold U, Vogelbacher G, Häring I, Riedel W (2014) Empirische Analyse sicherheitskritischer Ereignisse in urbanisierten Gebieten, Empirical analysis of security critical events in urban areas (in German). Bautechnik 92(4):262-273

Fischer K, Riedel W, Häring I, Nieuwenhuijs A, Crabbe S, Trojaborg S, Hynes W, Müllers I (2012) Vulnerability identification and resilience enhancements of urban environments. In: Aschenbruck N et al. (eds) 7th Security research conference, future security 2012. Springer, Communications in Computer and Information Science CCIS 318, Berlin, pp 176-179

Fischer K, Häring I, Riedel W, Vogelbacher G, Hiermaier S (2016) Susceptibility, vulnerability and averaged risk analysis for resilience enhancement of urban areas. Int J Protect Struct 7(1), 45-76

Fraunhofer EMI (2015) EU project VITRUV: vulnerability identification tools for resilience enhancements of urban environments. [cited 2016 14.07]. http://www.vitruv-project.eu

Füssel H-M (2007) Vulnerablility: a generally applicable conceptual framework for climate change research. Glob Environ Change 17(2):155-167

Gebbeken N, Döge T (2010) Explosion protection-architectural design, urban planning and landscape planning. Int J Protect Struct 1(1):1-21

Hardwick MJ, Hall J, Tatom JW, Baker RG (2009) Approved methods and algorithms for DoD risk based explosives siting. Department of Defence Explosives Safety Board, Alexandra

Häring I (2015) Risk analysis and management: engineering resilience. Springer, Singapore

Häring I, Ebenhöch S, Stolz A (2016a) Quantifying resilience for resilience engineering of socio technical systems. Eur J Secur Res 1(1):21-58

Häring I, Scharte B, Hiermaier S (2016b) Towards a novel and applicable approach for Resilience Engineering. In: 6-th International Disaster and Risk Conference (IDRC): Integrative Risk Management - towards resilient cities, Davos

Häring I, Scharte B, Stolz A, Leismann T, Hiermaier S (2016c) Resilience Engineering and Quantification for Sustainable Systems Development and Assessment: Socio-technical Systems and Critical Infrastructures. International Risk Governance Council (IRGC), Lausanne

Häring I, Schönherr M, Richter C (2009) Quantitative hazard and risk analysis for fragments of high explosive shells in air. Reliab Syst Saf Eng 94(9):1461-1470

Häring I, Gürke G (2006) Hazard densities for explosions. In: Soares CG, Zio E (eds) European safety and reliability conference (ESREL) 2006 of the European safety and reliability association (ESRA). CRC Press, Estoril, p 1987-1994

ISO (2009) ISO 31000: risk management-principles and guidelines. International Organization for Standardization, Geneva, pp 1-176 
Kaplan S, Garrick BJ (1981) On the quantitative definition of risk. Risk Anal 1(1):11-27

Kelling GL, Wilson JQ (1982) Broken windows: the police and neighborhood safety. In: The Atlantic Monthly 249(3):29-38

Kröger W, Zio E (2011) Vulnerable systems. Springer, London

Lin C-H, Liou D-Y, Wu K-W (2007) Opportunities and challenges created by terrorism. Technol Forecast Soc Change 74(2):148-164

Morgan MG, Henrion M (1992) Uncertainty: a guide to dealing with uncertainty in quantitative risk and policy analysis. Cambridge University Press, Cambridge

Proske D (2008) Catalogue of risks - natural, technical, social and health risks. Springer, Berlin Heidelberg

Richardson LF (1948) Variation of the frequency of fatal quarrels with magnitude. J Am Stat Assoc 43(244):523-546

Riedel W, Fischer K, Kranzer C, Erskine J, Cleave R, Hadden D, Romani M (2012) Modeling and validation of a wall-window retrofit system under blast loading. Eng Struct 37:235-245. doi:10. 1016/j.engstruct.2011.12.015

Riedel W, Fischer K, Stolz A, Häring I, Bachmann M (2015) Modeling the vulnerability of urban areas to explosion scenarios. In: Stewart MG, Netherton MD (eds) 3rd International conference on protective structures (ICPS3). Newcastle, Australia, pp 1-9

Riedel W, Niwenhuijs A, Fischer K, Crabbe S, Heynes W, Müllers I, Trojaborg S, Häring I (2014) Quantifying urban risk and vulnerability - a toolsuite of new methods for planners. In: Thoma K, Häring I, Leismann T (eds) 9th Future security research conference (future security). Fraunhofer, Berlin, pp 8-16

Salhab RG, Häring I, Radtke FKF (2011) Formalization of a quantitative risk analysis methodology for static explosive events. In: Bérenguer GGS, Soares G (eds) Advances in safety, reliability and risk management: European Safety and Reliability Association, European Safety and Reliability Conference (ESREL), CRC Press, Troyes, France, pp 1311-1320

Savitch HV (2005) An anatomy of urban terror: lessons from Jerusalem and elsewhere. Urban Studies 42(3):361-395

Siebold U, Ziehm J, Häring I (2009) Terror event database and analysis software. In: Future security, 4th security research conference, Fraunhofer, Karlsruhe

Siebold U, Häring I, Karwath A (2009) Statistical and time series analysis of terror event data for prediction and group spreading behavior (unpublished)

Smith PD (2010) Blast walls for structural protection against high explosive threats: a review. Int J Protect Struct 1(1):67-84

Smith K (2013) Environmental hazards: assessing risk and reducing disaster, 6 edn. Routledge, London

Stolz A, Fischer K, Roller C, Hauser S (2014) Dynamic bearing capacity of ductile concrete plates under blast loading. Int J Impact Eng 69:25-38

Taleb NN (2008) Der Schwarze Schwan: Die Macht höchst unwahrscheinlicher Ereignisse. Carl Hanser, München

Thoma K, Scharte B, Hiller D, Leismann T (2016) Resilience engineering as part of security research: definitions, concepts and science approaches. Eur J Secur Res 1(1):3-19

Thoma K (ed) (2014) Resilien-Tech: "Resilience-by-Design": Strategien für die technologischen Zukunfsthemen. acatech Studie, ed. G.N.A.o.S.a.T. (acatech), München: Herbert Utz Verlag

Turner BL, Kasperson RE, Matson PA, McCarthy JJ, Corell RW, Christensen L, Eckley N, Kasperson JX, Luers A, Martello ML, Poslsky C, Pulsipher A, Shiller A, (2003) A framework for vulnerability analysis in sustainability science. Proc Natl Acad Sci USA 100(14):8074-8079

UN/ISDR (2004) Living with risk: a global review of disaster reduction initiatives. United Nations InterAgency Secretariat of the International Strategy for Disaster Reduction (UN/ISDR), Editor 2004: Geneva

United Nations Department of Economic and Social Affairs (2014) World urbanization prospects, the 2014 revision, highlights, ed. United Nations, New York: United Nations

United Nations Development Programme (2015) Post-2015 Sustainable Development Agenda. [cited 2016 14.07], http://www.undp.org/content/undp/en/home/mdgoverview/post-2015-developmentagenda.html

van der Voort M, Radtke FKF, van Amselfort R, Khoe YS, Stacke I, Voss M, Häring I (2010) Recent developments of the KG software. 34-th Department of Defense Explosives Safety seminar 2010. Portland, Oregon, USA, pp 1-16 
Voss M, Häring I, Fischer K, Riedel W, Siebold U (2012) Susceptibility and vulnerability of urban buildings and infrastructure against terroristic threats from qualitative and quantitative risk analysis. In: IAPSAM \& ESRA (International Association for Probabilistic Safety Assessment and Management and European Safety and Reliability Association) (eds) 11th International probabilistic safety assessment and management conference and the annual european safety and reliability conference 2012 (PSAM11 ESREL 2012). Curran Associates, Inc., Helsinki, p 5757-5767

Willis HH, Morral AR, Kelly TK, Medby JJ (2005) Estimating terrorism risk. RAND Corporation, Center for Terrorism Risk Management Policy, Santa Monica, p 66

Woo G (2002) Quantitative terrorism risk assessment. J Risk Financ 4(1):7-14

Ziehm J, Häring I (2011) Risk analysis for a German harbour within the project ECSIT. In: Ender J, Fiege J (eds) 6th security research conference. Fraunhofer, Berlin, p 201-207

Ziehm J, Mayrhofer C, Häring I (2009) Scenario and damage analysis for terroristic attacks on a German airport. In: Elsner P (ed) 4th Future security research conference. Fraunhofer, Karlsruhe, p 261-271 